MONOGRÁFICO

SisTeMa HOSPITALARIO Y ENFERMERÍA EN LA España Moderna 



\title{
EREBEA
}

Revista de Humanidades

y Ciencias Sociales

Núm. 4 (2014), pp. 7-41

ISSN: 0214-0691

\section{LA HISTORIA DE LA ENFERMERÍA: ORIGEN Y DESARROLLO DE UNA LÍNEA DE INVESTIGACIÓN EN ESPAÑA (1989-2014)}

\author{
Antonio Claret García Martínez \\ Universidad de Huelva \\ Manuel Jesús García Martínez \\ Universidad de Sevilla
}

RESUMEN

Los hospitales son complejas instituciones que permiten una aproximación histórica desde diferentes perspectivas, lo que ha ido exigiendo la apertura de nuevas líneas de investigación. Este trabajo pretende mostrar las líneas de aproximación al mundo de los hospitales a través de los estudios desde la historia de la Enfermería o historia de los cuidados enfermeros, con una recopilación bibliográfica de más de 300 referencias, precedida de un estado de la cuestión que recoge su punto de partida.
ABstract

Hospitals are complex institutions that allow an historical approach from different perspectives, which has been allowing the opening of new lines of research. This paper aims to show the lines of approach to the world of hospitals across studies from nursing history or history of nursing care, with a bibliography of over 300 references, preceded by a state of the collecting and the starting point of this research.
Palabras Clave

Historia de la enfermería. Historia de los cuidados. Hospitales. Enfermería. Bibliografía.

\section{KeYwords}

Nursing history. Health cares history. Hospitals. Nursing. Bibliography. 



\section{INTRODUCCIÓN}

Hablar de Enfermería es hablar de cuidados, es decir, de personas que han adquirido una formación determinada para la prestación de cuidados de tipo sanitario en los hospitales y otros centros asistenciales, con el objetivo de ayudar a las personas a recuperar la salud perdida o a aliviar su sufrimiento.

En 1977 la Enfermería alcanzó en Espańa por primera vez en su historia el rango de estudio universitario a nivel de primer ciclo. Desde entonces, la profesión enfermera ha experimentado profundos cambios en sus planteamientos epistemológicos, científicos y profesionales, hasta el punto de quedar hoy perfectamente integrada en los estudios de Grado implantados en la Unión Europea tras el complejo proceso de Bolonia. Tal reconocimiento académico es el resultado de muchos siglos de evolución de una profesión, que existe como oficio desde tiempo inmemorial, aunque con unos perfiles poco definidos.

Esta elevación del nivel formativo de los profesionales de la Enfermería, tanto en el ámbito asistencial como en el docente, tuvo como consecuencia la toma de conciencia de los profesionales sobre la propia disciplina y propició el desarrollo de diversas líneas de investigación, entre ellas las que se ocupaban de la recuperación de la historia de la Enfermería española y su inserción en la Historia en sentido amplio, así como en la historia de la asistencia sanitataria en España en su sentido más concreto.

Todo este proceso implicóun aumento considerable de la investigación de la historia de la profesión enfermera en todo el territorio nacional, con la aparición de grupos de investigación, proyectos, reuniones científicas y un número creciente de publicaciones, que han cambiado por completo la visión que se tenía de la profesión hasta hace escasamente 25 años. Todo ello tiene una gestación y unos orígenes, cuyo conocimiento ayuda a explicar la situación actual de la investigación en historia de la Enfermería en España y su posición en el contexto internacional.

La Enfermería es una de las profesiones que han tenido a lo largo de la historia como singularidad la de contar con la presencia de la mujer en un altísimo porcentaje. Desde las sociedades más primitivas, el hecho del "cuidar" ha estado muy unido al entorno doméstico y a la mujer; con el establecimiento de instituciones basadas en la prestación de cuidados, ella ha venido a desempeñar un papel preponderante. Esto no quiere decir que el hombre haya quedado excluido 
sistemáticamente de las tareas cuidadoras, antes al contrario, desde el siglo XVI la fundación de congregaciones y órdenes dedicadas casi en exclusiva a la prestación de cuidados en los hospitales fueron promovidas por hombres, los cuales impulsaron el oficio, teorizaron sobre él y elaboraron tratados de enfermería que son hitos imprescindibles para conocer el desarrollo de la profesión en los últimos cinco siglos. ${ }^{1}$

\section{LOS INICIOS DE LA INVESTIGACIÓN}

Si realizáramos una búsqueda bibliográfica acerca de los trabajos publicados a comienzos de la década de los 80 de la pasada centuria sobre historia de la Enfermería en España, los registros obtenidos serían mínimos. Algunos estudios debidos a Carmen Domínguez Alcón, Francisco Ventosa Esquinaldo y Cecilio Eseverri Chaverri ${ }^{2}$ rompen el vacío existente, erigiéndose en pioneros o "precursores"; trabajos que sirvieron de referente en las escuelas de Enfermería del momento para introducir a los estudiantes en la historia de una profesión de la que se ingoraba prácticamente todo. ${ }^{3}$

\section{Período de CAMbios}

El panoramo comienza a cambiar en la segunda mitad de los años 80 , a causa del interés creciente suscitado en distintas instituciones vinculadas al entorno de la Enfermería, principalmente escuelas, pero también los colegios profesionales, ${ }^{4}$

1 Las Orden de San Juan de Dios y la Congregación de los enfermeros Obregones, fundadas en España en el siglo XVI, o la Congregación de los Bethlemitas, fundada en el siglo XVII en Guatemala, son exponenetes del impulso que la Enfermería experimentó a comienzos de la Edad Moderna. Puede verse al respecto A. C. García Martínez, M. J. García Martínez, J. I. Valle Racero, "Un siglo de Oro para la Enfermería española (1550-1650): II. Desarrollo de las Funciones específicas de Enfermería”. En F. Hernández Martín (Coord.): Historia de la Enfermería en España (desde la Antigüedad hasta nuestros dias). Madrid:Editorial Síntesis, S. A., 1996, pp. 149-176;

M. J. García Martínez, "Cuidados enfermeros en la España del siglo XVII. Hacia la búsqueda de una identidad profesional”. Gazeta de Antropología, núm. 20. Granada: 2004. Publicación electrónica. Disponible en: http://www.ugr.es/-pwlac/G20 22ManuelJesus Garcia Martinez.html; M. J. García Martínez, J. I. Valle Racero, A. C. García Martínez, "Las funciones de enfermería en los Hermanos Obregones (siglos XVI-XVIII). Enfermería y Administración”. Escuela Universitaria de Enfermería, Fisioterapia y Podología (Universidad Complutense de Madrid), Escuela Universitaria de Enfermería del Hospital General Universitario de Valencia, Libro de Ponencias y Comunicaciones del I Congreso Nacional de Historia de la Enfermería, celebrado en Valencia. Barcelona: Fundación Uriach 1838, 1996, pp. 149-158.

2 C. Alcón, Los cuidados y la profesión enfermera en España, Madrid: Ediciones Pirámides S.A., 1986. C. Eseverri Chaverri, Historia de la Enfermería Española e Hispanoamericana. Salvat Editores, S.A. Barcelona, 1984.; F. Ventosa Esquinaldo, Historia de la Enfermería Española, Madrid: Editorial Ciencia, 1984.

3 Los estudios sobre historia de la Enfermería en las escuelas universitarias se completaba fundamentalmente con los estudios de historia de la Medicina y otros de historia de las Ciencias.

4 Desde hace más de veinte años una línea de investigación muy frecuentada ha sido el estudio 
los hospitales ${ }^{5}$ y el Consejo General de Enfermería de España. ${ }^{6}$ Todos ellos, ya fuese a nivel de la institución o, más frecuentemente, por iniciativa de los profesionales adscritos a ellas, sobre todo los profesores universitarios, comenzaron a organizar actividades que tenían como eje central la investigación y difusión de la historia de la profesión enfermera. Por otra parte, las revistas enfermeras de base generalista, con preponderancia de estudios clínicos, incluían cada vez con más frecuencia breves artículos de contenido histórico, que daban tímidamente a conocer los resultados de ese interés por los temas relacionados con el pasado de la profesión. ${ }^{7}$ Entre todas ellas habría que destacar Índex de Enfermería, publicación con sede en Granada que ha mostrado desde su fundación una especial sensibilidad hacia los temás históricos y humanísticos en general. ${ }^{8}$

\section{LA ECLOSIÓN DE LOS ESTUDIOS SOBRE HISTORIA DE LA ENFERMERÍA}

En este contexto hay que resaltar las actividades promovidas desde la Escuela de Enfermería de la Universidad Complutense de Madrid, bajo la dirección de Francisca Hernández Martín, Profesora Titular de Historia de la Enfermería. La

de la historia de los colegios profesionales de Enfermería. Fundados desde finales del siglo XIX, los colegios profesionales han desempeñado un papel de primera importacia en el desarrollo de la profesión, con el planteamiento sistemático de reivindicaciones profesionales y de lucha por el colectivo enfermero. Los archivos que guardan muchos de ellos ha posibilitado la realización de estudios sobre ellos. Puede verse al respecto para el Colegio Oficial de Enfermería de Sevilla C. Gallardo Moraleda, E. Jaldón García, V. Villa García-Noblejas, La Enfermería sevillana. El Colegio y su Historia (1900/1930). Granada: Ilustre Colegio Oficial de Enfermería de Sevilla, 1993; y C. Gallardo Moraleda, V. Villa García-Noblejas, La Enfermería sevillana. El Colegio y su Historia (1961/1990). Sevilla: Ilustre Colegio Oficial de Enfermería de Sevilla, 2002.

5 El sistema hospitalario español ha experimentado una profunda renovación en el siglo XX, buscando la modernización e incorporación de las tecnologías que este siglo ha ido proporcionando. Esto se ha traducido en la renovación de antiguas instalaciones y, sobre todo, en la construcción de nuevos complejos hospitalarios. Con una edad de entre sesenta y ochenta años, muchos hospitales españoles de última generación tienen ya tras de sí una historia que contar y lo están empezando a hacer, creciendo en los últimos años las conmemoraciones y estudios monográficos sobre ellos.

6 El Consejo General de Enfermería de España es el órgano que agrupa a todos los Consejos autonómicos de Enfermería y a todos los Colegios Oficiales de Enfermería de España. Constituye un órgano cuyos orígenes se remontan a finales del siglo XIX y que experimentó un importante desarrollo a partir de los años 70 y 80 del siglo pasado. Dentro de su línea de publicaciones ha incluido trabajos de investigación histórica, desde la edición de fuentes hasta monografías.

7 Con carácter nacional cabe destacar Rol. Revista de Enfermería, que se edita desde 1979; o, a nivel provincial, Hygia. Revista Cientifica del Colegio Oficial de Enfermería de Sevilla, editada desde los años ochenta. Éstas y otras incluían de manera más o menos frecuente breves artículos de tipo histórico, y sirvieron para hacer visible una investigación en continuo crecimiento. De similares características a las anteriores, aunque dedicada al ámbito de las matronas (especialidad actual de la Enfermería) es Matronas hoy, promovida por la Asociación Nacional de Matronas desde comienzos de los años noventa.

8 Index de Enfermería. Gaceta Bibliográfica del Centro de Documentación en Enfermería Comunitaria. Granada.Su primer número fue publicado en 1992. 
realización de seminarios especializados, la convocatoria de un Premio Internacional de Historia de la Enfermería, la puesta en marcha del Seminario Permanente para la Investigación de la Historia de la Enfermería y la organización de Jornadas científicas supondrían hitos históricos de consecuencias trascendentales para comprender, en gran medida, la evolución de los estudios sobrehistoria de la profesión. Paralelamente, fueron apareciendo investigadores y grupos de investigación en distintos puntos del país, que comenzaron una tarea de búsqueda, localización, estudio y edición de las fuentes históricas, que enriquecieron el panorama historiográfico sobre la materia. ${ }^{?}$

Así, desde Granada, con la publicación Índex de Enfermería (promovida por la Fundación Índex desde 1992), desde Sevilla-Huelva con la puesta en marcha de Hiades. Revista de Historia de la Enfermería (1994), primera publicación periódica monográfica, ${ }^{10} \mathrm{y}$ desde el Departamento de Enfermería de la Universidad de Alicante, con su publicación Cultura de los Cuidados, ${ }^{11}$ se genera un amplio movimiento de investigación que aglutinó a investigadores de todo el país, publicando en sus números el avance de la investigación. Desde Madrid, Valladolid, Barcelona, Málaga, Cádiz, Valencia, Guipúzcoa y desde otras muchas ciudades han ido incorporándose investigadores que, en su conjunto, han conseguido construir una visión más amplia y real de la evolución de la historia de los cuidados en España.

En todo este panorama hay que destacar la puesta en marcha desde el Seminario Permanente para la Investigación de la Historia de la Enfermería (dirigido por la Profesora Francisca J. Hernández Martín, de la Universidad Complutense) de los Congresos Nacionales e Internacionales de Historia de la Enfermería. En 1992 y 1993 se celebraron las Primeras y Segundas Jornadas de Historia de la Enfermería, ${ }^{12}$

9 Sería difícil recoger en unas pocas líneas los investigadores que a lo largo de los años han ido incorporándose a la investigación histórica de la profesión, no quedando en la actualidad ninguna comunidad autónoma sin que disponga de grupos o investigadores a título individual que lo hagan.

10 Hiades. Revista de Historia de la Enfermería lleva 11 números publicados hasta 2014 y en ellos han publicado un total de más de 300 investidores desde su fundación en 1994. Véase http:// www.portalhiades.com

11 El Departamento de Enfermería de la Universidad de Alicante ha desempeñado un papel de primera importancia en el desarrollo de la Enfermería Universitaria desde los años 80, y de especial relevancia en la investigación de la historia de la Enfermería. Profesores como José Siles González, Catedrático de Enfermería, han desarrollado su labor consiguiendo la implantación del Título Propio de Enfermería, el Doctorado y finalmente el Grado. Desde su Cátedra, ha dirigido tesis doctorales sobre Historia de la Enfermería y ha luchado por incluir en los estudios universitarios de Enfermería la Historia como asignatura. Ha promovido Jornadas y Congresos y fundado la Asociación de Historia y Antropología de los Cuidados, que mantiene estrechas relaciones con profesores y alumnado de las más importantes universidades hispanoamericanas, promoviendo desde 1997 Cultura de los Cuidados. Revista de Enfermería y Humanidades.

12 La celebración de las I Jornadas Nacionales de Investigación en Historia de la Enfermería bajo el título de "La Enfermería en el siglo XX: de oficio a profesión" celebradas en Madrid en octubre 
que se transformaron a partir de 1994 en congresos nacionales. El primero, celebrado en Valencia en 1994, a los que siguieron los de Málaga, Mérida, Gijón y Sevilla. El $V$ Congreso Nacional de Historia de la Enfermería celebrado en Sevilla significó un punto de inflexión. La participación de investigadores procedentes de Brasil, Colombia, México y otros países favoreció el proceso de reflexión y la necesidad de avanzar un poco más en la organización de estos eventos científicos, pasando a tener desde entonces carácter de internacional a partir del sexto; las siguientes sedes fueron Alcalá de Henares, Granada, Zaragoza, Santa Cruz de Tenerife, Almería, Barcelona, Alicante y Madrid. En la actualidad, se está organizando el XIV Congreso Nacional y IX Internacional de Historia de la Enfermería en Santader, para mayo de 2015.

Desde 1989 hasta 2014 son 25 años en los que el panorama bibliográfico ha cambiado por completo. Los Colegios Profesionales de Enfermería están realizando una interesante labor de investigación de su propia historia, que muchos de ellos pueden remotarla hasta finales del siglo XIX; los hospitales, las escuelas de Enfermería, el Consejo General y otras instituciones han desarrollado una labor muy interesante para la recuperación de la historia de la profesión.

LA INVESTIGACIÓN DE LA HISTORIA DE LA ENFERMERÍA EN EL CONTEXTO INTERNACIONAL

El interés por la investigación de la historia de la Enfermería no se circunscribe exclusivamente al ámbito español, ocurriendo un proceso similar a nivel internacional. Un punto de referencia obligado lo marca el inicio del interés por esta investigación en la Universidad de Pensilvania, en los Estados Unidos. En los años ochenta de la pasada centuria comienzan a tomar forma algunas instituciones que vendrían a ocupar un lugar de primera importancia en este panorama con el paso de los años. Así, se funda en esta universidad norteamericana el Barbara Bates Center for the Study of the History of Nursing, institución que servirá de referencia internacional sobre los estudios de historia de la Enfermería. Paralelamente, en los ańos noventa y en la nueva centuria surgen grupos de investigadores en distintos países europeos e iberoamericanos, como Brasil, Colombia, Chile y México, por citar a los más activos, pero también en Europa, en Portugal, el Reino Unido, Dinamarca, Francia, Italia y Alemania, igualmente por citar los centros demás proyección. Toda esta actividad está generando la formación de asociaciones internacionales, como la European Association for the History of NursingEAHN), fundada en 2012 y que agrupa a asociaciones de 13 países europeos. Por otra

de 1992 suponen un hito en el desarrollo de los estudios en historia de la profesión. Estas Jornadas y los congresos subsiguientes sirivieron para aglutinar a un número creciente de investigadores, planificar estrategias, promover eventos científicos y motivar la reflexión sobre el pasado de la profesión. Resultado de ellas, en 2015 se celebrará el XIV Congreso Nacional y IX Internacional de Historia de la Enfermería. 
parte, la investigación de la historia de la Enfermería recibió un importante reconocimiento en el año 2012 al ser incluida oficialmente como una de las líneas presentes en las convocatorias cuadrianuales del Congreso que organiza el Consejo Internacional de Enfermería, fundado en 1899, y que en ese año se celebró en la isla de Malta.

\section{LA ACTUALIDAD}

Hoy podemos afirmar sin temor a equivocarnos que se cuentan por miles las referencias bibliográficas generadas en este terreno y hay que plantearse la posibilidad de llevar a cabo una publicación que recoja la mayor parte de la misma. Incluimos en Erebea una síntesiscon el avance de este nuevo espacio de investigación, que se ha ido abriendo camino en los últimos 25; un dominio bantante desconocido en el ámbito historiografía histórica más general, al haberse desarrollado muy en el interior de la propia disciplina enfermera. Creemos que, por su importancia, volumen y calidad debe ser conocida por los especialistas en temas sanitarios, de la historia de los hospitales y de otros espacios de investigación con el que la Enfermería guarda una vinculación muy estrecha.

La presente recopilación bibliográfica pretende difundir, por tanto, las principales líneas de investigación abiertas en los últimos años en entorno a la historia de la Enfermería española.

\section{LA PRESENTE RECOPILACIÓN BIBLIOGRÁFICA}

Siempre resulta difícil proponer una selección bibliográfica;con frecuencia se quedan fuera títulos importantes, otras veces son incluidos otros que podían haber sido obviados. Sin embargo, este tipo de trabajo se hace necesario porque posiblitan a muchos interesados establecer un primer contacto con una materia que desconocen; en otras ocasiones, algunos investigadores amplían el conocimiento que sobre ella ya tenían. A pesar de sus luces ysombras, una recopilación bibliográfica nos acerca siempre a líneas de investigación, a temas o planteamientos nuevos de una realidad ya existente; a nuevas lecturas sobre viejas fuentes, en definitiva, a espacios de reflexión que están llevando a cabo un grupo amplio de investigadores. Casi nunca es fácil organizar los títulos con unos criterios claros y definidos; muchos trabajos participan de distintos espacios de investigación, imbricándose unos con otros,porque todos formanparte de una misma realidad. No es fácil analizar el trabajo de las enfermeras de forma completamente aislada de su entorno físico (el hospital) y humano (médicos, cirujanos, boticarios y otros profesionales, sanitarios o no). Sin embargo, con frecuencia observamos que se han realizado muchos estudios de corte médico en los ambientes hospitalarios en donde la presencia de los cuidadores o enfermeros se ha visto sensiblemente reducida, y no necesarimente por una intención declarada; posiblemente, las fuentes consultadas no han sido siempre las más idóneas o completas. 
Por ello, las secciones que hemos establecido en esta recopilación bibliográfica está enfocada a dirigir al interesado hacia las grandes áreas temáticas que han recibido la atención de los historiadores de la Enfermería, subrayando una vez más la ineludible interrelación entre todas ellas. En concreto, son seis:

- Historia de la profesión enfermera.

- Hospitales, Instituciones y Personajes relacionados con el cuidado.

- Investigación y Metodología en Historia de la Enfermería.

- El trabajo enfermero a lo largo de la historia: funciones, tareas y cuidados.

- Fuentes para la historia de la Enfermería.

- Publicaciones periódicas con mayor presencia de la historia de la Enfermería.

- Libros de Actas de Congresos de historia de la Enfermería celebrados en España.

Las más de 300 referencias incluidas han de ser consideradas como una muestra de la amplia relación de publicaciones existentes en nuestro país o, en caso de las referencias internacionales, consultadas de forma más asidua por los especialistas. ${ }^{13}$

13 En estos momentos estamos llevando a cabo un recopilación bibliográfica sobre historia de la Enfermería a nivel internacional, que podrá ofrecer al lector una visión de conjunto de las principales líneas de investigación que sobre la materia se están desarrollando a nivel internacional. 
Historia de LA PROFESIÓN ENFERMERA

- M. Alpañez Pardo, Historia moderna de la Enfermería española (siglos XIX-XX). Almería: Autor-editor, 2007.

- C. C. Álvarez Nebreda, Semblanzas de la profesión enfermera (1862 - 1953). Madrid: Colegio Oficial de Enfermería de Madrid, 2011.

- J. A. Ávila Olivares, "Los precursores oficiales de los practicantes de mitad del siglo XIX: los sangradores”. Hiades. Revista de Historia de la Enfermería, n. ${ }^{\circ} 11$ (2014).

- J. Bernabeu Mestre, E. Gascón Pérez, Historia de la Enfermería de Salud Pública en España (1860-1977). Murcia: Publicaciones de la Universidad de Alicante, 1999.

- A. Buendía Muñoz, I. García Morales, La Enfermería en la Almería de los siglos XVIII y XIX. Cuidados y cuidadores. Sevilla: Colegio de Enfermería de Almería, 2003.

- M. a E. Bulcão, M. Mendonça, M. M. a Silva Vieira, "A formação das parteiras em Portugal -de 1836 a 1967-“. Hiades. Revista de Historia de la Enfermería, n. ${ }^{\circ} 11$ (2014).

- M. Cabré, T. Ortiz (Eds.), Sanadoras, matronas y médicas en Europa. Siglos XIIXX. Barcelona: Icaria Editorial, S. A., 2001.

- R. Camaño Puig, "Proceso de profesionalización: evolución de la denominación de Enfermería”. Hiades. Revista de Historia de la Enfermería, n. 10 (2008), pp. 903-920.

- L. C. Casado Marín, M. ${ }^{a}$ J. Montes Muñoz, "El savoir faire/saber hacer de las matronas durante el Nacional-Catolicismo en España”. Hiades. Revista de Historia de la Enfermería, n. ${ }^{\circ} 11$ (2014).

- E. Castro Manrique, "Orígenes de la Enfermería y sus antecedentes históricos en la Universidad de Valladolid (de la Ley Moyano a la II República, 18571936)". Enfermería Clinica no 4 (5).

- Colegio Oficial de Enfermería de Gipuzkoa, Apuntes históricos de Gipuzkoa. Practicantes, Matronas y Enfermeras (194 - 2004). San Sebastián, 2006.

- C. Domínguez-Alcón, La Infermeria a Catalunya. Barcelona: Ediciones Rol, S. A., 1981.

- C. Domínguez-Alcón, J. M.a de Miguel, J. M. Rodríguez, Sociología y Enfermería. Madrid:Ediciones Pirámide, 1983.

- C. Domínguez-Alcón, Los cuidados y la profesión enfermera en España. Madrid:Ediciones Pirámide, 1986.

- C. Domínguez-Alcón, "Demandas sociales de salud a lo largo de la historia: motor de cambio de la praxis infermera". Hiades. Revista de Historia de la Enfermería, n. ${ }^{o} 11$ (2014).

- M. P. Donahue, Historia de la Enfermería. Barcelona: Editorial Doyma, 1988. 
- F. M. Elizondo, Desarrollo histórico de la Enfermería. México: Prensa Médica Mexicana, 1965.

- C. Eseverri Chaverri, Historia de la Enfermería Española e Hispanoamericana. Madrid: Editorial Univérsitas, S. A., 1995.

- Escuela de Enfermería y Fisioterapia, La enfermería profesional. Libro de Actas. VI congreso Nacional y I Internacional de Historia de la Enfermería. Navarra: Servicio de Publicaciones de la Universidad de Alcalá, 2003.

- M. ${ }^{a}$ L. Fernández, J. L. Callejo, "Historia de la alimentación. Algunas consideracions desde la Historia de la Enfermería". Hiades. Revista de Historia de la Enfermería, n. ${ }^{\circ} 10$ (2008), pp. 513-538.

- L. Fernández Donaire, La Historia de la Enfermería en el contexto del Espacio Europeo de Educación Superior. Barcelona: Servicio de Publicaciones de la Universidad Autónoma de Barcelona, 2008.

- M. ${ }^{a}$ C. Fernández Mérida, "Matronas y asociacionismo". En Álvarez Nebreda, Carlos C. y Hernández Martín, F.a J. (editores), El asociacionismo en la Enfermería y su influencia en el desarrollo de la profesión: 100 años de historia del Colegio de Enfermeras de Madrid (1862-2012). Madrid: Colegio Oficial de Enfermería de Madrid, 2013, pp. 255-264.

- J. Ferreira, O. Ferreira, H. Silva, "O ensino da história de enfermagem na Escola Superior de Enfermagem de Lisboa (Esel)". Hiades. Revista de Historia de la Enfermería, n.o 11 (2014).

- M. Ferreiro Ardions, J. Lazaún Valdubieco, Historia de la Enfermería en Álava. Álava: Colegio Oficial de Enfermería de Álava, 2008.

- M. Ferreiro Ardions, J. Lazaún Valdubieco, "Los Ilustrados alaveses en la pugna entre matronas y cirujanos durante el siglo XVIII". Hiades. Revista de Historia de la Enfermería, n. ${ }^{\circ} 1$ (2014).

- C. García Martín-Caro, M. ${ }^{a}$ L. Martínez Martín, Historia de la Enfermería: evolución histórica del cuidado enfermero. Barcelona: Elsevier España, S. A., 2010.

- A. C. García Martínez, "Enfermeras laicas en el Madrid de los siglos XVII y XVIII. ¿Vocación o Profesión? Hiades. Revista de Historia de la Enfermería, n. ${ }^{\circ} 11$ (2014).

- A. C. García Martínez, M. J. García Martínez, J. I. Valle Racero, "Génesis de la Enfermería Moderna en España”, en F. Hernández Martín (coord.): Historia de la Enfermería en España (desde la Antigüedad hasta nuestros días). Madrid: Editorial Síntesis, S. A., 1996, pp. 123-136.

- A. C. García Martínez, y otros, "Fechas claves de la Enfermería”. Híades. Revista de Historia de la Enfermería, n. 1 (agosto de 1994), pp, 63-68.

- A. C. García Martínez, M. J. García Martínez, "Fechas claves para la historia de las matronas en España". Hiades. Revista de Historia de la Enfermería, n. ${ }^{\circ}$ 5-6 (1999), pp, 243-260.

- A. C. García Martínez, M. J. García Martínez, “Aportación de las cuidadoras a 
la asistencia del mundo occidental". En Carmen González Canalejo y Fernando Martínez López (Eds.), La transformación de la Enfermería. Nuevas miradas para la Historia. Granada: Editorial Comares, S. L., 2010, pp. 117-134.

- M. J. García Martínez, "Cuidados enfermeros en la España del siglo XVII. Hacia la búsqueda de una identidad profesional". Gazeta de Antropología, n. 20 (2004), texto 20-22.

- M. J. García Martínez, "Historia del arte de los partos en el ámbito familiar. En Cultura de los Cuidados, n. 24 (2. ${ }^{\circ}$ semestre de 2008), pp. 40-47.

- M. J. García Martínez, "El asociacionismo enfermero. Reflexiones desde la Historia de la profesión”. En Álvarez Nebreda, Carlos C. y Hernández Martín, F. ${ }^{a}$ J. (editores), El asociacionismo en la Enfermeria y su influencia en el desarrollo de la profesión: 100 años de historia del Colegio de Enfermeras de Madrid (1862-2012). Madrid: Colegio Oficial de Enfermería de Madrid, 2013, pp. $153-172$.

- M. García Pardo, "La mujer y la asistencia sanitaria en la ciudad de Almería (siglos XV-XVI)". En C. González Canalejo, F. Martínez López (editores), La transformación de la Enfermería. Nuevas miradas para la Historia. Granada: Editorial Comares, 2010, pp. 95-116.

- M. ${ }^{a}$ del C. García Velázquez, "Perfil profesional de la enfermera española a lo largo de la Historia". Hiades. Revista de Historia de la Enfermería, n. ${ }^{\circ} 10$ (2008), pp. 977-984.

- D. Garriga Guitart y otros, Del Bimaristán al hospital psiquiátrico: historia de la enfermería y la salud mental en el Islam. Madrid: Asociación de Enfermería en Salud Mental, 2010.

- T. Geovanini, A. Moreira, S. Dornelles, W. C. A. Machado, História da Enfermagem. Versōes e interpretaçóes. Rio de Janeiro: Livraria e Editora Revinter Ltda. Terceira Ediçao, 2010.

- C. González Canalejo, F. Martínez López (editores), La transformación de la Enfermería. Nuevas miradas para la Historia. Granada: Editorial Comares, 2010.

- C. González Canalejo, "Mujeres sanitarias y derechos de ciudadanía en la España contemporánea”. En C. González Canalejo, F. Martínez López (editores), La transformación de la Enfermería. Nuevas miradas para la Historia. Granada: Editorial Comares, 2010, pp. 163.182.

- M. ${ }^{a}$ E. González Iglesias, "Los inicios de la Enfermería como profesión". En Álvarez Nebreda, Carlos C. y Hernández Martín, F.a J. (editores), El asociacionismo en la Enfermería y su influencia en el desarrollo de la profesión: 100 años de historia del Colegio de Enfermeras de Madrid (1862-2012). Madrid: Colegio Oficial de Enfermería de Madrid, 2013, pp. 265-274.

- J. Hernández Conesa, Historia de la Enfermería. Un análisis histórico de los cuidados de Enfermería. Madrid:Interamericana McGraw-Hill, 1995.

- F. Hernández Martín (Coord.): Historia de la Enfermería en España (desde la 
Antigüedad hasta nuestros días). Madrid:Editorial Síntesis, S. A., 1996.

- B. Hurtado Pardos, C. Nebot-Bergua, "Historia de los cuidados de enfermería en las unidades de cuidados intensivos pediátricos y neonatales". Hiades. Revista de Historia de la Enfermería, n. ${ }^{\circ} 11$ (2014).

- I. Jiménez Rodríguez, "Algunas consideraciones sobre el origen y evolución de los Practicantes”. En Álvarez Nebreda, Carlos C. y Hernández Martín, F. J. (editores), El asociacionismo en la Enfermería y su influencia en el desarrollo de la profesión: 100 años de historia del Colegio de Enfermeras de Madrid (18622012). Madrid: Colegio Oficial de Enfermería de Madrid, 2013, pp. 173-196. - M. a López Vallecillo, “Formación de enfermeras en 1942”. Hiades. Revista de Historia de la Enfermería, n. ${ }^{\circ} 1$ (2014).

- M. a. Martínez Martín, Historia de la Enfermería: evolución histórica del Cuidado enfermero. Barcelona: Elsevier España, S. A., 2. ${ }^{\text {a ed., }} 2011$.

- F. Martínez Bruque, M. Pérez Luna, Historia de la Enfermería en Badajoz.Del Potobarbeirato a la Enfermería universitaria. Badajoz: Editora Regional de Extremadura. Servicio de Publicaciones de la UEX, 2005.

- A. Martínez Molina, "Desarrollo profesional de la Matrona a través de su historia”. En Álvarez Nebreda, Carlos C. y Hernández Martín, F. a J. (editores), El asociacionismo en la Enfermería y su influencia en el desarrollo de la profesión: 100 años de historia del Colegio de Enfermeras de Madrid (1862-2012). Madrid: Colegio Oficial de Enfermería de Madrid, 2013, pp. 197-215.

- C. Negrillo Durán, "Evolución y desarrollo de los cuidados femeninos y masculinos en la historia de la Enfermería". Hiades. Revista de Historia de la Enfermeria, n.o 10 (2008), pp. 453-465.

- T. Oguisso, M. ${ }^{\mathrm{a}}$ J. Schmidt, $O$ Exercício da Enfermagem. Uma abordagem éticoLegal. Rio de Janeiro: Editora Guanabara Koogan, S. A., 2010.

- T. Oguisso, P. F. de Souza Campos, "La Enfermería del siglo XX en Brasil y su aporte al bienestar social”. En C. González Canalejo, F. Martínez López (editores), La transformación de la Enfermería. Nuevas miradas para la Historia. Granada: Editorial Comares, 2010, pp. 351-360.

- M. ${ }^{a}$ C. Ortego Maté, "Enfermería profesión. De ATS a Diplomado en Enfermería (1953-2002)". Rol. Revista de Enfermería, vol. 25, n. 9 (septiembre de 2002), pp. 16-21.

- T. Ortiz, "Protomedicato y matronas. Una relación al servicio de la cirugía”. Revista Dynamis, n. 16 (1996), pp. 109-120.

- J. Parrilla Saldańa, "La Enfermería en el Siglo XX. De oficio a profesión. Evolución de la Enfermería en Sevilla. Siglo XX”. Madrid: E. U. Enfermería, Fisioterapia y Podología. Universidad Complutense de Madrid (1995), pp. 111-116.

- J. Parrilla Saldaña, "La Enfermería en los Siglos XVIII-XIX. Cuidados de enfermería en la Beneficencia española. Siglos XVIII-XIX. Madrid: E. U. Enfer- 
mería, Fisioterapia y Podología. Universidad Complutense de Madrid (1995), pp. 121-138.

- J. Parrilla Saldaña, "La Enfermería en la ruta jacobea. Perspectiva histórica. Historia y orígenes de la Orden Militar de Santiago y de la Ruta Jacobea". Híades. Revista de Historia de la Enfermería, n. ${ }^{\circ} 8$ (2001), pp. 259-266.

- J. Parrilla Saldaña, "La Enfermería profesional. Análisis de la Enfermería en España desde finales del siglo XIX hasta su integración en la Universidad". Madrid: Universidad de Alcalá de Henares, 2003, pp. 37-38.

- M. Pedrosa Cuenca, Historia de la Enfermería. Málaga: 2008.

- M. ${ }^{a}$ J. Pereira Pinilla, "Implicación de la Enfermería en las Campañas de Marruecos". Hiades. Revista de Historia de la Enfermería, n. ${ }^{\circ} 11$ (2014).

- F. Porto, W. Amorim (organizadores), História da Enfermagem. Identidade, profissionalização e simbolos. São Paulo (Brasil): Yendis Editora Ltda. São Caetano do Sul, 2010.

- F. Porto, W. Amorim (organizadores), História da Enfermagem Brasileira. Lutas, Ritos e Emblemas. Rio de Janeiro: Editora Águia Dourada Ltda., 2007.

- M. a J. Pujol y otros, "La Escuela Nacional de Puericultura y sus enfermeras visitadores". Hiades. Revista de Historia de la Enfermería, n. ${ }^{o} 11$ (2014).

- A. Ramió Jofre, "Valores sociales y valores de Enfermería a través de la Historia". Híades. Revista de Historia de la Enfermería, n.o 11 (2014).

- M. ${ }^{a}$ A. Rodríguez Rozalén, "Matronas: ¿Especialidad enfermera o profesión?”. En Álvarez Nebreda, Carlos C. y Hernández Martín, F.a J. (editores), El asociacionismo en la Enfermería y su influencia en el desarrollo de la profesión: 100 años de historia del Colegio de Enfermeras de Madrid (1862-2012). Madrid: Colegio Oficial de Enfermería de Madrid, 2013, pp. 239-254.

- M. ${ }^{a}$ D. Ruiz-Berdún, "El reconocimiento de la colegiación de las matronas". En Álvarez Nebreda, Carlos C. y Hernández Martín, F.a J. (editores), El asociacionismo en la Enfermería y su influencia en el desarrollo de la profesión: 100 años de historia del Colegio de Enfermeras de Madrid (1862-2012). Madrid: Colegio Oficial de Enfermería de Madrid, 2013, pp. 217-238.

- M. Santo Tomás Pérez, "Historia de la Enfermería". En Enfermería Fundamental. Barcelona: Masson, 1994.

- M. Santo Tomás Pérez, "Pugna por el reconocimiento universitario de la Enfermería y su especialización”. En Álvarez Nebreda, Carlos C. y Hernández Martín, F.a J. (editores), El asociacionismo en la Enfermería y su influencia en el desarrollo de la profesión: 100 años de historia del Colegio de Enfermeras de Madrid (1862-2012). Madrid: Colegio Oficial de Enfermería de Madrid, 2013, pp. 297-332.

- J. M. de los Santos Alfonso, "Del cuidado natural a la Enfermería Profesional". Hiades. Revista de Historia de la Enfermería, n. o 5-6 (1999), pp. 75-91.

- M. ${ }^{a}$ C. Sellán Soto y otros, La profesión va por dentro: elementos para una historia 
de la Enfermería española contemporánea. Madrid: FUDEN, 2. ${ }^{\text {a }}$ ed., 2010.

- J. Siles González, Pasado, presente y futuro de la Enfermería en España. Alicante: CECOVA, 1996.

- J. Siles González, Historia de la Enfermería. Alicante: Editorial Aguaclara. Colección Amalgama, 1999.

- J. Siles González, Historia de la Enfermería. Madrid: Ediciones DAE (Grupo Paradigma), 2011.

- J. Siles González, T. Oguisso, G. Fernandes de Freitas, P. F. Souza Campos (compiladores), Cultura de los Cuidados: Historia de la Enfermería iberoamericana. Alicante: Editorial Club Universitario, 2011.

- M. Solórzano Sánchez, Apuntes históricos de Gipuzcoa. Practicantes, Matronas y Enfermeras 1904-2004. San Sebastián: Diputación Foral de Gipuzcoa, 2007.

- J. Sousa, H. Neves, I. Rocha, M. Maia, "El Contexto Socio-Político en el desarrollo de la Enfermería en Portugal". Hiades. Revista de Historia de la Enfermería, n. ${ }^{\circ} 11$ (2014).

- T. Urien Blázquez, Enfermería: Historia desde 1857. Sevilla: Editorial Visión Libros, 2011.

- J. I. Valle Racero, A. C. García Martínez, M. J. García Martínez, "Génesis de la Enfermería Moderna en España”. En F. Hernández Martín (Coord.): Historia de la Enfermería en España (desde la Antigüedad hasta nuestros dias). Madrid:Editorial Síntesis, S. A., 1996, pp. 123-136.

- F. Ventosa Esquinaldo, Historia de la Enfermería española. Madrid: Ciencia 3. Distribución, S. A., 1984.

- F. Ventosa Esquinaldo, Cuidados psiquiátricos en enfermería en España-siglos XV al XX-. Una aproximación histórica. Madrid: Ediciones Díaz de Santos, S. A., 2000.

- F. Ventosa Esquinaldo, "Pugna por la unificación de los Practicantes, Matronas y Enfermeras en una sola profesión"”. En Álvarez Nebreda, Carlos C. y Hernández Martín, F.a J. (editores), El asociacionismo en la Enfermería y su influencia en el desarrollo de la profesión: 100 años de historia del Colegio de Enfermeras de Madrid (1862-2012). Madrid: Colegio Oficial de Enfermería de Madrid, 2013, pp. 133-152.

- M. Vigil, La vida de las mujeres en los siglos XVI y XVII. Madrid: Siglo Veintiuno de España Editores, S. A., 1986. 
Hospitales, Institucionesy Personajes relacionados con el cuidado

- C. C. Álvarez Nebreda, "150 años de historia del Colegio de Enfermeras de Madrid: motor de la organización colegial de España”. En Álvarez Nebreda, Carlos C. y Hernández Martín, F.a J. (editores), El asociacionismo en la Enfermería y su influencia en el desarrollo de la profesión: 100 años de historia del Colegio de Enfermeras de Madrid (1862-2012). Madrid: Colegio Oficial de Enfermería de Madrid, 2013, pp. 35-60.

- C. Álvarez, S. Dacal, J. M. Lama, M. a A. Rodríguez, "Gaucelmo y San Fructuoso, dos bercianos en el Camino". Hiades. Revista de Historia de la Enfermería, n. ${ }^{\circ} 10$ (2008), pp. 1185-1190.

- A. Arroyo Rodríguez, F. J. Alemay Lasheras, R. Romero Serrano, M. ${ }^{\text {a S. Morillo }}$ Martín, I. Lancarro Tavero, "Evolución de los Registros de Enfermería en los Hospitales de la Orden Hospitalaria de San Juan de Dios de Sevilla”. Híades. Revista de Historia de la Enfermería, n. ${ }^{\circ} 11$ (2014).

- V. M. Backes, "El legado histórico del modelo Nightingale: su estilo de pensamiento y praxis". Horizonte de Enfermería, año IX, n. ${ }^{\circ} 1$ (1998), Universidad Federal de Santa María (Brasil), pp. 7-21.

- P. Barreiro Pereira, J. M. Bermejo, El Colegio de Enfermería de Madrid. Cien años de Historia. Madrid: Colegio Oficial de Enfermería de Madrid, 2009.

- S. Bartoloni, "La asistencia a los enfermos en Italia: religiosa y laicas en la práctica de la Enfermería”. En C. González Canalejo, F. Martínez López (editores), La transformación de la Enfermería. Nuevas miradas para la Historia. Granada: Editorial Comares, 2010, pp. 151-162.

- M. A. Calvo Calvo, "La reacción de los colegios oficiales de Practicantes a la creación de la enfermera titulada en 1927”. En Álvarez Nebreda, Carlos C. y Hernández Martín, F.a J. (editores), El asociacionismo en la Enfermería y su influencia en el desarrollo de la profesión: 100 años de historia del Colegio de Enfermeras de Madrid (1862-2012). Madrid: Colegio Oficial de Enfermería de Madrid, 2013, pp. 383-390.

- R. Camaño-Puig, "Perfil de Salud en un hospital del siglo XIX: Hospital de San Miguel y Na Marcena (Sagunto, España)". Hiades. Revista de Historia de la Enfermería, n. 10 (2008), pp. 591-598.

- J. I. Carmona García, El sistema de la hospitalidad pública en la Sevilla del Antiguo Régimen. Sevilla: Diputación Provincial de Sevilla, 1979.

- M. ${ }^{a}$ I. Coelho de Souza, V. Regina Salles, "A mística do silencio. A prática de Enfermagem na Santa Casa de Misericórdia do Rio de Janeiro no século XIX". Hiades. Revista de Historia de la Enfermería, n. ${ }^{\circ} 10$ (2008), pp. 491-512.

- C. B. Collado, "Influencia de las organizaciones internacionales de la salud en la evolución de la Enfermería”. En Álvarez Nebreda, Carlos C. y Hernández Martín, F.a J. (editores), El asociacionismo en la Enfermería y su influencia en el 
desarrollo de la profesión: 100 años de historia del Colegio de Enfermeras de Madrid (1862-2012). Madrid: Colegio Oficial de Enfermería de Madrid, 2013, pp. 61-87.

- Consejo de Enfermería de la Comunidad Valenciana (CECOVA), 1986-2011. 25 Aniversario. Alicante: CECOVA, 2011.

- C. Eseverri Chaverri, Juan de Dios, el de Granada. Granada: Editorial La Vela, 2001.

- C. Eseverri Chaverri, "Enfermería vallisoletana. Siglo XVI, antes y después. El Hospital Rio Hortega, Valladolid". Hiades. Revista de Historia de la Enfermería, n. 10 (2008), pp. 345-352.

- R. Expósito González, "Organización y lucha por el reconocimiento de los Colegios de Practicantes”. En Álvarez Nebreda, Carlos C. y Hernández Martín, F. ${ }^{a}$ J. (editores), El asociacionismo en la Enfermería y su influencia en el desarrollo de la profesión: 100 años de historia del Colegio de Enfermeras de Madrid (1862-2012). Madrid: Colegio Oficial de Enfermería de Madrid, 2013, pp. 113-132.

- M. ${ }^{a}$ L. Fernández, J. L. Callejo, B. Arizaga, M. Santo Tomás, "La red hospitalaria y asilar de Cantabria en la ruta de la costa del Camino de Santiago". Hiades. Revista de Historia de la Enfermería, n.o 8 (2001), pp. 223-234.

- M. ${ }^{a}$ L. Fernández Fernández, S. Fernández Moral, "La famosa escritora Vera Brittain, enfermera en la I Guerra Mundial”. En C. González Canalejo, F. Martínez López (editores), La transformación de la Enfermería. Nuevas miradas para la Historia. Granada: Editorial Comares, 2010, pp. 183-202.

- M. ${ }^{a}$ C. Fernández Mérida, "La función del personal de enfermería en el Hospital Provincial de Málaga en el siglo XIX según el Reglamento de 1861". Hiades. Revista de Historia de la Enfermería, n. ${ }^{\circ} 10$ (2008), pp. 583-590.

- M. ${ }^{a}$ C. Fernández Mérida, "Enfermería en el siglo XVII. Hospital de Bubas de Santa Ana de Málaga”. En C. González Canalejo, F. Martínez López (editores), La transformación de la Enfermería. Nuevas miradas para la Historia. Granada: Editorial Comares, 2010, pp. 277-294.

- O. Ferreira, "O Projecto de uma Escola de Enfermeiras de Saúde Pública no Portugal de 1930". Hiades. Revista de Historia de la Enfermería, n. 11 (2014).

- M. Fraile Bravo, F. Tirado Altamirano, L. M. Hernández Neila, J. Prieto Moreno, "SEAPEX: Germen de Asociación Científica de Atención Primera". En Álvarez Nebreda, Carlos C. y Hernández Martín, F.a J. (editores), El asociacionismo en la Enfermería y su influencia en el desarrollo de la profesión: 100 años de historia del Colegio de Enfermeras de Madrid (1862-2012). Madrid: Colegio Oficial de Enfermería de Madrid, 2013, pp. 463-468.

- M. E. Galiana Sánchez, J. Bertomeu Mestre, M. P. García Paramio, "Enfermeras para una nueva patria. Género e ideología en la Enfermería de Falange". En C. González Canalejo, F. Martínez López (editores), La transformación de 
la Enfermería. Nuevas miradas para la Historia. Granada: Editorial Comares, 2010, pp. 203-218.

- C. Gallardo Moraleda, E. Jaldón García, V. Villa García-Noblejas, La Enfermería sevillana. El Colegio y su Historia (1900/1930). Granada: Ilustre Colegio Oficial de Enfermería de Sevilla, 1993.

- C. Gallardo Moraleda, V. Villa García-Noblejas, La Enfermería sevillana. El Colegio y su Historia (1961/1990). Sevilla: Ilustre Colegio Oficial de Enfermería de Sevilla, 2002.

- S. García Barrios, "El Hospital de Beneficencia Provincial de San Juan de Dios de Málaga en los siglos XIX y XXo”. En C. González Canalejo, F. Martínez López (editores), La transformación de la Enfermería. Nuevas miradas para la Historia. Granada: Editorial Comares, 2010, pp. 325338.

- S. García Barrios, M. ${ }^{a}$ T. Labajos Manzanares, P. Rojero Anaya, "La Guerra Civil en Málaga y el Hospital Civil Provincial (1936-1939)".Hiades. Revista de Historia de la Enfermería, n. ${ }^{\circ} 11$ (2014).

- A. C. García Martínez, "Asociaciones para la asistencia social: Los cuidados infantiles en la Casa Cuna de Sevilla a través de sus reglas de 1656". En Álvarez Nebreda, Carlos C. y Hernández Martín, F.a J. (editores), El asociacionismo en la Enfermería y su influencia en el desarrollo de la profesión: 100 años de historia del Colegio de Enfermeras de Madrid (1862-2012). Madrid: Colegio Oficial de Enfermería de Madrid, 2013, pp. 491-497.

- A. C. García Martínez, M. J. García Martínez, "El enfermero obregón Andrés Fernández: un impulsor de la Enfermería española en la primera mitad del siglo XVII". Hiades. Revista de Historia de la Enfermería, n.o 11 (2014).

- A. C. García Martínez y otros, "El modelo de Enfermería de San Juan de Dios". Index de Enfermería, n. ${ }^{\circ} 14$ (1995), pp. 31-35.

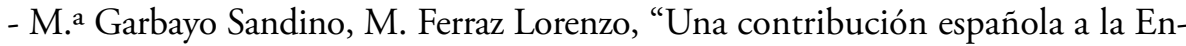
fermería y Pedagogía en la América Hispana del siglo XVII: el canario Pedro de Betancur". Hiades. Revista de Historia de la Enfermería, n. ${ }^{\circ} 10$ (2008), pp. 379-406.

- C. Germán Bes, Historia de la institución de la Enfermería universitaria: análisis con una perspectiva de género. Zaragoza: Prensas Universitarias de Zaragoza, 2007.

- M. ${ }^{a}$ C. Giménez Muñoz, Los establecimientos benéficos más relevantes de Sevilla hasta 1849. Sevilla: Ediciones Alfar, 2008.

- R. Gómez, J. Garrido, E. de la Peña, S. Galeano, "La Casa de Dementes de mediados del siglo XIX en la Ciudad de Mérida". Hiades. Revista de Historia de la Enfermería, n. ${ }^{\circ} 10$ (2008), pp. 639-644.

- M. ${ }^{a}$ E. González Iglesias, R. M. a Prol Cid, J. Siles González, "Los cuidados de enfermería en el Ourense de la primera mitad del siglo XX. Las Siervas de María, Ministras de los Enfermos”. En C. González Canalejo, F. Martínez López 
(editores), La transformación de la Enfermería. Nuevas miradas para la Historia. Granada: Editorial Comares, 2010, pp. 339.350.

- M. ${ }^{a}$ D. Guerra, V. Fernández, "Perspectiva histórica de un centro de salud desde el punto de vista de una enfermera". Hiades. Revista de Historia de la Enfermería, n. ${ }^{\circ} 10$ (2008), pp. 677-682.

- J. M.a Hernández Conesa, "El asociacionismo tridentino: de los hospitales y de los enfermos". En Álvarez Nebreda, Carlos C. y Hernández Martín, Faa J. (editores), El asociacionismo en la Enfermería y su influencia en el desarrollo de la profesión: 100 años de historia del Colegio de Enfermeras de Madrid (18622012). Madrid: Colegio Oficial de Enfermería de Madrid, 2013, pp. 333-338.

- F. J. Hernández Martín, "Luisa de Marillac: precursora de la Enfermería Moderna". Hiades. Revista de Historia de la Enfermería, n. 11 (2014).

- F. Hernández Martín, R. del Gallego Lastra, "Virginia Henderson, autora clave para la Enfermería actual". Hiades. Revista de Historia de la Enfermería, n. o 5-6 (1999), pp. 11-23.

- F. Hernández Martín, M. Morente, "Definir la Enfermería desde la Historia: Humberto de Romans y el oficio de enfermero". Hiades. Revista de Historia de la Enfermería, n. ${ }^{\circ} 10$ (2008), pp. 271-282.

- H. Hernández Martínez, M. ${ }^{a}$ I. Pascual Benito, 40 años de la Escuela Universitaria de Enfermería de Guadalajara, 1968/69-2008/09. Alcalá de Henares (Madrid): Servicio de Publicaciones de la Universidad de Alcalá de Henares, 2009.

- M. Herrero y Rodríguez de Miñón, "Las organizaciones corporativas y su función social y profesional”. En Álvarez Nebreda, Carlos C. y Hernández Martín, F. J. (editores), El asociacionismo en la Enfermería y su influencia en el desarrollo de la profesión: 100 años de historia del Colegio de Enfermeras de Madrid (1862-2012). Madrid: Colegio Oficial de Enfermería de Madrid, 2013, pp. $29-34$.

- C. Lozano Peña, "Las mujeres en los establecimientos benéficos de Granada en la Edad Moderna”. En C. González Canalejo, F. Martínez López (editores), La transformación de la Enfermería. Nuevas miradas para la Historia. Granada: Editorial Comares, 2010, pp. 135-150.

- B. Madarieta Revilla y otros, "Exposición Temporal: Florence Nightingale, Maestra en el Arte de Cuidar". Hiades. Revista de Historia de la Enfermería, n. ${ }^{\circ}$ 11 (2014).

- S. Malchau Dietz, Women Religious and Nursing in the Renaissance. The Daughters of Charity and the Professionalization of Nursing. Kolding (Dinamarca): The Danish Museum of Nursing History. University of Southern Denmark, 2010.

- N. Martín Espinosa, "La prestación de cuidados en los establecimientos reunidos de Toledo a principios del siglo XX: una cuestión de fe". Híades. Revista de Historia de la Enfermería, n. ${ }^{o} 11$ (2014). 
- L. Martínez Rodríguez, "Pasado y futuro de la espiritualidad en enfermería desde la Experiencia de San Juan de Dios". Hiades. Revista de Historia de la Enfermería, n. ${ }^{\circ} 11$ (2014).

- M. ${ }^{a}$ D. Mayán, S. Puyó, "La historia de la Escuela de Enfermeras de Santa Madrona a través de la fotografia: 1917-1936". Hiades. Revista de Historia de la Enfermería, n. ${ }^{\circ} 10$ (2008), pp. 569-582.

- F. Nightingale, Letters of Florence Nightingale in the history of nursing archive. Boston: University of Boston, 1974.

- F. Nightingale, Notes on hospital. Nueya York: Gordon, 1976.

- A. Nogales Espert, E. Llorca Marqués, "Avances en Historia de la Enfermería desde los hospitales renacentistas (Valencia, siglos XV-XVII)”. En C. González Canalejo, F. Martínez López (editores), La transformación de la Enfermería. Nuevas miradas para la Historia. Granada: Editorial Comares, 2010, pp. 273276.

- A. Nogales Espert, "Apuntes sobre el Hospital de San Lázaro de Valencia, de 1484-85 y 1485-86". Hiades. Revista de Historia de la Enfermería, n. 10 (2008), pp. 623-638.

- T. Ortiz, "De matrona a matrona: Francisca Iracheta y la divulgación de la ciencia obstétrica en España en 1870". Arenal, n. ${ }^{\circ} 6$ (1), enero-junio de 1999, pp. 183-195.

- J. M. a Palanca y otros, "Estructura de los hospitales leoneses del siglo XVI. A propósito del Hospital de Nuestra Señora la Blanca de Puente Villarente". Hiades. Revista de Historia de la Enfermería, n. 10 (2008), pp. 609-616.

- J. Parrilla Saldaña, "Metodología e investigación en historia de la Enfermería. Los hospitales de Sevilla en la Baja Edad Media". Hiades. Revista de Historia Enfermería, n. ${ }^{\circ}$ 5-6 (marzo de 1998), pp. 103-114.

- F. J. Prieto Miguélez, "Asturica Augusta: Ciudad hospitalaria”. Hiades. Revista de Historia de la Enfermería, n. 10 (2008), pp. 599-608.

- J. Prieto, L. M. Hernández, F. Tirado, A. Moreno, "Los Reales Hospitales y Enfermería de Santa María de Guadalupe". Hiades. Revista de Historia de la Enfermería, n. 10 (2008), pp. 645-652.

- J. Prieto, L. M. Hernández, F. Tirado, A. Moreno, "Funcionamiento de los Reales Hospitales de Guadalupe: funciones de enfermería". Híades. Revista de Historia de la Enfermería, n. 10 (2008), pp. 653-660.

- J. Prieto, L. M. Hernández, F. Tirado, A. Moreno, "Enfermeros y médicos ilustres de los Reales Hospitales de Guadalupe". Hiades. Revista de Historia de la Enfermería, n.o 10 (2008), pp. 661-669.

- J. Prieto Moreno, L. M. Hernández Neila, F. Tirado Altamirano, M. Fraile Bravo, "Los inicios del Colegio de Enfermería de Cáceres". En Álvarez Nebreda, Carlos C. y Hernández Martín, F. J. (editores), El asociacionismo en la Enfermería y su influencia en el desarrollo de la profesión: 100 años de historia 
del Colegio de Enfermeras de Madrid (1862-2012). Madrid: Colegio Oficial de Enfermería de Madrid, 2013, pp. 613-618.

- M. ${ }^{a}$ R. Ribas Poch, "Margaret Sanger, enfermera pionera en la lucha a favor del control de la natalidad". Hiades. Revista de Historia de la Enfermería, n. ${ }^{\circ} 11$ (2014).

- S. del Río, M. a M. Pérez, E. Calvo, S. García, "Historia del Hospital de Antequera: Hospital de Santa Ana (siglo XVI, 1667)". Hiades. Revista de Historia de la Enfermeria, n. ${ }^{\circ} 10$ (2008), pp. 669-676.

- M. Rodríguez, P. Fernández, R. Martínez, M. ${ }^{a}$ A. Rodríguez, "Los hospitales: El refugio de los peregrinos en el Bierzo". Hiades. Revista de Historia de la Enfermería, n. 10 (2008), pp. 695-720.

- J. Sánchez Martínez, Kénôsis-Diakonia en el itinerario espiritual de San Juan de Dios. Granada: Archivo-Museo San Juan de Dios "Casa de los Pisa". Colección Temas Históricos O. H., 2014.

- M. Solórzano Sánchez, Historia y antecedentes del Hospital de Amara. San Sebastián: CHDO, 1999.

- M. Solórzano Sánchez, Dispensario Médico de Santa Isabel. Gratuito para los pobres de San Sebastián. San Sebastián: Hospital Donostia, 2002.

- M. Solórzano Sánchez, 50 años del Hospital Domostia. San Sebastián: Hospital Donostia, 2010.

- M. Solórzano Sánchez, Hospital Civil de San Antonio Abad. 50 años de la desaparición del primer hospital de San Sebastián. San Sebastián: Osakidetza, 2011.

- M. Solórzano Sánchez, "Alexis Soyer: el colaborador de Florence Nightingale enCrimera, Jesús Rubio Pilarte". Hiades. Revista de Historia de la Enfermería, n. ${ }^{\circ} 11$ (2014).

- E. Souto do Nascimento, V. Mascarenhas Nascimento Teixeira, "Escola de Enfermagem Hugo Werneck: Traços de uma história". Hiades. Revista de Historia de la Enfermería, n. ${ }^{\circ} 11$ (2014).

- M. Süsskind Borenstein, "O cotidiano da Enfermagem no Hospital de Caridade de Florianòpolis -SC (Brasil), no período de 1935 a 1968”. Hiades. Revista de Historia de la Enfermería, n. 10 (2008), pp. 539-550.

- C. Torres Penella, A. Ramió Jofre, "Memoria de una Dama de Sanidad Militar: Carmen Bolea Foradada”. En Álvarez Nebreda, Carlos C. y Hernández Martín, F.a J. (editores), El asociacionismo en la Enfermería y su influencia en el desarrollo de la profesión: 100 años de historia del Colegio de Enfermeras de Madrid (1862-2012). Madrid: Colegio Oficial de Enfermería de Madrid, 2013, pp. 659-666.

- R. Valls, Ma C. Olivé, A. Pardo, "La evolución històrica de la Escuela de Enfermería de la Universidad de Barcelona". Hiades. Revista de Historia de la Enfermería, n. ${ }^{\circ} 10$ (2008), pp. 617-622.

- F. Ventosa Esquinaldo, "San Juan de Dios". Hiades. Revista de Historia de la 
Enfermeria, n. ${ }^{\circ} 11$ (2014).

- F. Ventosa Esquinaldo, Pensamiento de San Juan de Dios y la Orden Hospitalaria y su relación con la Enfermería: conceptos y valores. Granada: Archivo-Museo San Juan de Dios "Casa de los Pisa”. Colección Temas Históricos O. H., 2012.

- C. Vila Gimeno, "Nota sobre la historia del servicio de urgencias del Hospital de San Pau de Barcelona (1967-1980)". Hiades. Revista de Historia de la Enfermería, n. ${ }^{\circ} 11$ (2014).

\section{InVestigación y Metodología en Historia de la Enfermería}

- R. Alberdi Castell, "Los contextos conceptuales históricos: una propuesta de análisis de la evolución de la profesión enfermera desde los orígenes hasta el siglo XIX". Hiades. Revista de Historia de la Enfermería, n. 5-6 (1999), pp. 63-73.

- C. P. Arredondo González y otros, "Museo Histórico de Enfermería. Un espacio virtual para el Aprendizaje, la Reflexión y la Investigación". Hiades. Revista de Historia de la Enfermería, n. ${ }^{\circ} 1$ (2014).

- J. A. Ávila Olivares, Evolución histórica del movimiento colegial de Enfermería: el Colegio de Alicante. Tesis doctoral. Alicante: Universidad de Alicante, 2011.

- J. Cabrero García, M. Richart Martínez, Investigar en Enfermería. Concepto y estado actual de la investigación en Enfermería. Alicante: Universidad de Alicante, 2001.

- M. ${ }^{a}$ G. Cid Expósito, I. Jiménez Rodríguez, I. Corral Liria, "El futuro inmediato como parte de la asignatura de Historia de la Enfermería en el Pregrado". En Álvarez Nebreda, Carlos C. y Hernández Martín, F.a J. (editores), El asociacionismo en la Enfermería y su influencia en el desarrollo de la profesión: 100 años de historia del Colegio de Enfermeras de Madrid (1862-2012). Madrid: Colegio Oficial de Enfermería de Madrid, 2013, pp. 431-436.

- M.a G. Cid Expósito, I. Jiménez Rodríguez, I. Corral Liria, "Metodología enfermera en un manicomio del siglo XV". En Álvarez Nebreda, Carlos C. y Hernández Martín, F.a J. (editores), El asociacionismo en la Enfermería y su influencia en el desarrollo de la profesión: 100 años de historia del Colegio de Enfermeras de Madrid (1862-2012). Madrid: Colegio Oficial de Enfermería de Madrid, 2013, pp. 437-444.

- F. G. Conde Mora, "El archivo histórico de la Asociación Salus Infirmorum de Cádiz: un referente para la investigación de la Historia de la Enfermería”. En Álvarez Nebreda, Carlos C. y Hernández Martín, F.a J. (editores), El asociacionismo en la Enfermería y su influencia en el desarrollo de la profesión: 100 años de historia del Colegio de Enfermeras de Madrid (1862-2012). Madrid: Colegio Oficial de Enfermería de Madrid, 2013, pp. 445-450.

- R. Daza Caballero, "Genealogía: método de la enseñanza de la Historia”. Hía- 
des. Revista de Historia de la Enfermería, n. ${ }^{o} 10$ (2008), pp. 931-936.

- P. Galbany Estragués, A. Ramió Jofre, R. Valls Molins, A. Guilera Roche, "Recuperar la memoria histórica para hacer visible la Enfermería: historias de vida dedicadas a la profesión”. Hiades. Revista de Historia de la Enfermería, n. ${ }^{1} 1$ (2014).

- A. C. García Martínez, "Del CD-ROM a Internet. Los nuevos soportes para la investigación histórica”. Hiades. Revista de Historia de la Enfermería, n. 9 (2002/2003), pp. 43-56.

- A. C. García Martínez, "Cultura escrita y grupos profesionales. La escritura y la lectura entre los enfermeros españoles de los siglos XVI y XVII". Historia. Instituciones. Documentos, n. 31 (2004), pp. 249-267.

- A. C. García Martínez, J. I. Valle Racero, M. J. García Martínez, "Las funciones de enfermería en los Hermanos Obregones (siglos XVI-XVIII). Docencia e Investigación”. Escuela Universitaria de Enfermería, Fisioterapia y Podología (Universidad Complutense de Madrid), Escuela Universitaria de Enfermería del Hospital General Universitario de Valencia, Libro de Ponencias y Comunicaciones del I Congreso Nacional de Historia de la Enfermeria, celebrado en Valencia. Barcelona: Fundación Uriach 1838, 1996, pp. 99-107.

- M. J. García Martínez, "El método en la Enfermería. Una mirada desde la Historia (siglo XVII)". Metas de Enfermería, vol. 7, n. ${ }^{\circ} 10$ (2004/2005), pp. 58-64.

- M. J. García Martínez, Cuidar el cuerpo y salvar las almas: La práctica de la Enfermería según el modelo de la Congregación de enfermeros Obregones. Tesis doctoral. Director: Dr. Salvador Rodríguez Becerra. Sevilla: Servicio de Publicaciones de la Universidad de Sevilla, 2007. Disponible en: http://www. fondosdigitales.us.es/tesis/1616

- C. González Canalejo y otros, "Análisis de los aspectos metodológicos y humanísticos en la Enfermería actual". Cultura de los Cuidados. Revista de Enfermería y Humanidades, n.o 4 (1998), pp. 40-44.

- C. González Canalejo, "Metodología y Fuentes para el estudio de la Historia de la Enfermería Contemporánea”. Hiades. Revista de Historia de la Enfermería, n. ${ }^{\circ} 10$ (2008), pp. 879-888.

- J. D. González Sanz, "La orientación docente en Historia de la Enfermería: retos y dificultades en la ReformaProtestante". Hiades. Revista de Historia de la Enfermeria, n. ${ }^{\circ} 11$ (2014).

- M. Gordon, "Pasado, presente y futuro de los diagnósticos de Enfermería". Cultura de los Cuidados, n. 7 y 8 (1. ${ }^{\circ}$ y 2. ${ }^{\circ}$ semestres de 2000), pp. 128-138.

- F. a J. Hernández Martín, "Balance de dos décadas (1988-2008): contribución de la Historia de la Enfermería a la historiografía española”. En C. González Canalejo, F. Martínez López (editores), La transformación de la Enfermería. Nuevas miradas para la Historia. Granada: Editorial Comares, 2010, pp. 499-510. 
- F. Herrera Rodríguez, "Reflexiones sobre Historia de la Enfermería". EnDiez años de Enfermería universitaria en Cádiz. Cádiz: Servicio de Publicaciones de la Universidad de Cádiz, 1989, pp. 57-61.

- M. ${ }^{a}$ A. Huertas Ortas, Investigación en la historia de la Enfermería. Málaga: Monsálvez 2007 S. L., 2010.

- M. A. Martínez y otros, "Las Sutilezas de la Escritura en las Realidades de la Historia". Hiades. Revista de Historia de la Enfermería, n. ${ }^{\circ} 10$ (2008), pp. 969-976.

- A. Nogales Espert, "El proceso histórico de la Enfermería: origen y consecuencia del Pensamiento Enfermero". Hiades. Revista de Historia de la Enfermería, n. ${ }^{\circ}$ 10 (2008), pp. 937-943.

- A. Nogales Espert, "Investigación en Historia de la Enfermería y su aportación al crecimiento professional". Hiades. Revista de Historia de la Enfermería, n. ${ }^{\circ}$ 11 (2014).

- A. Nogales Espert, "Evolución histórica del Método Enfermero". Cultura de los Cuidados. Revista de Enfermería y Humanidades, n.o 13 (2003), pp. 23-28.

- T. Oguisso, P. F. Souza Campos, G. Fernandes de Freitas (organização), Pesquisa em História da Enfermagem. São Paulo (Brasil): Editora Manole Ltda, 2011.

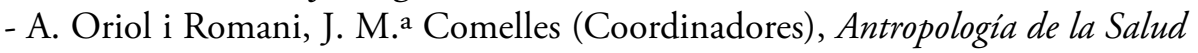
$y$ de la Medicina. Tenerife:Actas del VI Congreso de Antropología. Volumen 2, 1993.

- J. Sánchez García y otros, "Proceso de cuidados desde el modelo enfermero de Virginia Henderson: caso clínico". Revista Metas de Enfermería, año III, n. ${ }^{\circ}$ 25 (2000), pp. 36-41.

- P. Sanchiz Ochoa, "El Archivo de Indias y la Antropología Histórica". Jiménez Núñez, A. (comp.), Antropología histórica: La Audiencia de Guatemala en el siglo XVI. Sevilla: Secretariado de Publicaciones de la Universidad de Sevilla, 1997, pp. 53-60.

- M. Santo Tomás Pérez, "Líneas de investigación para avanzar en la Historia de la Enfermería”. En C. González Canalejo, F. Martínez López (editores), La transformación de la Enfermería. Nuevas miradas para la Historia. Granada: Editorial Comares, 2010, pp. 393-402.

- I. Santos y otros, "(Re)construindo uma nova história de Enfermagem”. Híades. Revista de Historia de la Enfermería, n. 10 (2008), pp. 985-996.

- M. ${ }^{a}$ J. Sanz Fuentes, "Diplomática y Enfermería en Sevilla. Cirujanos y examinadores de cirujanos". Hiades. Revista de Historia de la Enfermería, n. ${ }^{\circ} 10$ (2008), pp. 189-202.

- C. Sellán, A, Vázquez, F. Blanco, "Cuerpos, apariencia y poder. Una odisea iconográfica a través de la Enfermería española contemporánea (1896-1977)”. En C. González Canalejo, F. Martínez López (editores), La transformación de la Enfermería. Nuevas miradas para la Historia. Granada: Editorial Comares, 2010, pp. 447-468. 
El trabajo ENFERMERo a lo largo de la Historia: FUnCiones, tareas y CUIDADOS

- I. M. a Aguilera, M. Rodríguez, "La Gota de Leche onubense: la asistencia y el control del niño sano en la primera mitad del siglo XX". Hiades. Revista de Historia de la Enfermería, n. 10 (2008), pp. 465-476.

- E. Alarcón, "Las prácticas de cuidados en las sociedades prehistóricas: la cultura argárica”. Arqueología y Territorio n.o 4 (2007), pp. 223-249.

- M. P. Álvaro, A. Carrero, M. J. Morlans, M. P. Fernández, "Prácticas de salud tradicionales y populares". Hiades. Revista de Historia de la Enfermería, n. 10 (2008), pp. 551-568.

- M. Blasco, J. Cía, P. Cía, "El oficio de enfermero en la Orden de San Jerónimo a través de sus Historias Generales". Hiades. Revista de Historia de la Enfermería, n. ${ }^{\circ} 10$ (2008), pp. 683-694.

- A. Buendía, I. García, “QQué dolor de muelas! Barberos, sangradores y practicantes. El cuidados de las enfermedades dentarias en la Almería de los siglos XVIII y XIX”. Hiades. Revista de Historia de la Enfermeria, n. ${ }^{\circ} 10$ (2008), pp. 413-426.

- M. A. Calvo Calvo, "Aspectos socio-laborales de los enfermeros del Hospital del Espíritu Santo de Sevilla a finales del siglo XVI". Hiades. Revista de Historia de la Enfermeria, n. 8 (2001), pp. 355-380.

- M. L. Canal, M. J. Morlans, P. Álvaro, J. D. Pedrera, "La atención a los ancianos en la Regla de San Fructuoso del Bierzo". Hiades. Revista de Historia de la Enfermeria, n. 8 (2001), pp. 245-249.

- A. Delgado Marchante, "La alimentación en la Ruta Jacobea”. Estudio introductorio y edición de la obra. Hiades. Revista de Historia de la Enfermería, n. ${ }^{o}$ 8 (2001), pp. 131-148.

- C. Domínguez-Alcón, Los cuidados y la profesión enfermera en España. Madrid:Ediciones Pirámide, 1986.

- A. Castillo Fernández, N. Gutiérrez Malax-Echevarría, "Cuidados sociosanitarios de la cultura Hebrea”. En Álvarez Nebreda, Carlos C. y Hernández

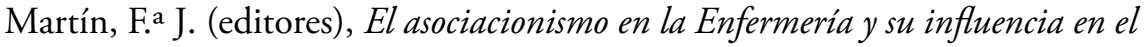
desarrollo de la profesión: 100 años de historia del Colegio de Enfermeras de Madrid (1862-2012). Madrid: Colegio Oficial de Enfermería de Madrid, 2013, pp. 415-424.

- M. Celma Vicente, "Cuidadoras informales y enfermeras. Relaciones dentro del hospital”. Revista Rol de Enfermería, año XXV, n.o 3 (2003), pp. 22-30.

- M. ${ }^{a}$ F. Collière, Promover la vida. De la práctica de las mujeres cuidadoras a los cuidados de enfermería. Madrid: McGraw-Hill. Interamericana, 1997.

- A. C. García Martínez, "La Enfermería franciscana en el siglo XVIII: Recetario Medicinal espagírico (1713), de Diego Bercebal”. Estudio introductorio y 
edición de la obra. Hiades. Revista de Historia de la Enfermería, n. ${ }^{\circ} 10$ (2008), pp. 655-774.

- A. C. García Martínez, M. J. García Martínez, J. I. Valle Racero, "Un siglo de Oro para la Enfermería española (1550-1650): II. Desarrollo de las Funciones específicas de Enfermería”. En F. Hernández Martín (Coord.): Historia de la Enfermería en España (desde la Antigüedad hasta nuestros dias). Madrid:Editorial Síntesis, S. A., 1996, pp. 149-176.

- A. C. García Martínez, M. J. García Martínez, "Las funciones de la matrona en el mundo antiguo y medieval. Una mirada desde la Historia". Matronas Profesión, volumen 6, núm. 1 (2005), pp. 11-18.

- M. J. García Martínez, "Prácticas ancestrales de las matronas espańolas: el agua de socorro o bautismo de urgencia". Hiades. Revista de Historia de la Enfermería, n. ${ }^{\circ} 11$ (2014).

- M. J. García Martínez, "Cuidados enfermeros en la España del siglo XVII. Hacia la búsqueda de una identidad profesional". Gazeta de Antropología, núm. 20. Granada: 2004. Publicación electrónica. Disponible en: http://www.ugr.es/ - pwlac/G20_22ManuelJesus_Garcia_Martinez.html

- M. J. García Martínez, "El Capítulo X de las Constituciones manuscritas elaboradas por Bernardino de Obregón: un nuevo concepto de Enfermería a finales del siglo XVI". Hiades. Revista de Historia de la Enfermería, n. ${ }^{\circ} 10$ (2008), pp. 255-269.

- M. J. García Martínez, J. I. Valle Racero, A. C. García Martínez, "Las funciones de enfermería en los Hermanos Obregones (siglos XVI-XVIII). Enfermería y Administración”. Escuela Universitaria de Enfermería, Fisioterapia y Podología (Universidad Complutense de Madrid), Escuela Universitaria de Enfermería del Hospital General Universitario de Valencia, Libro de Ponencias y Comunicaciones del I Congreso Nacional de Historia de la Enfermería, celebrado en Valencia. Barcelona: Fundación Uriach 1838, 1996, pp. 149-158.

- M. J. García Martínez, F. de Llanos Peña,"La ética del cuidar en la Enfermería española del siglo XVII: El manual para la formación de los enfermeros de Simón López (año 1668)". Libro de Actas de las IX Jornadas Nacionales de Bioética. Jerez de la Frontera: Orden Hospitalaria de San Juan de Dios, 2002, pp. 269-296.

- M. J. García Martínez, A. C. García Martínez, "Las funciones asistencial y docente de la partera en los siglos XVI y XVII. Su reflejo en el tratado Libro del parto humano". Híades. Revista de Historia de la Enfermería, n. 10 (2008), pp. 581-605.

- R. Gómez, E. de la Peńa, J. Garrido, S. Galeano, "Cuidados generales para mantener una vida sana en la España del siglo I d. C.”. Hiades. Revista de Historia de la Enfermería, n. ${ }^{\circ} 10$ (2008), pp. 477-483.

- A. González García, " La representación de la Enfermería a través de la labor 
sanitaria de la sección femeninaen las campañas de vacunación obligatoria de postguerra". Hiades. Revista de Historia de la Enfermería, n. ${ }^{\circ} 11$ (2014).

- J. Lezaun Valdubieco, M. Ferreiro Ardións, "Iglesia y lactancia artificial en los siglos XVIII y XIX: de la Animalidad alRegeneracionismo Moral”. Hiades. Revista de Historia de la Enfermería, n. ${ }^{\circ} 11$ (2014).

- S. López, Directorio de Enfermeros y artifice de obras de caridad para curar las enfermedades del cuerpo. Biblioteca de Clásicos de la Enfermería Espańola, n. ${ }^{\circ}$ 1. Estudio, trascripción e índices: Antonio C. García Martínez y Manuel J. García Martínez. Sevilla: Consejo General de Enfermería de España, 2001.

- C. Lozano Peña, "La jornada laboral de un enfermero del siglo XVIII". Híades. Revista de Historia de la Enfermería, n. ${ }^{\circ} 8$ (2001), pp. 407-416.

- F. de Llanos Peña, M. J. García Martínez, "Hacia la conformación de una ética profesional: las tareas de ayuda al bien morir en los manuales de enfermería de los Hermanos Enfermeros Obregones (siglos XVI-XVIII)". Libro de Actas de las VIII Jornadas Nacionales y IV Internacionales de Bioética. Sevilla: Orden Hospitalaria de San Juan de Dios, 2001, pp. 331-347.

- L. Martínez García, La asistencia a los pobres en Burgos en la Baja Edad Media. Burgos: Diputación Provincial de Burgos, 1981.

- A. Martínez Molina, Estudio histórico de la Enfermería en España. Vol. I: "La profesión de la Matrona según el Tratado de Damiá Carbó, siglo XVI”. Valencia: Fundación de Enfermería Internacional, 1994.

- A. Martínez Molina, Estudio histórico de la Enfermería en España. Vol. II: "La profesión de la Matrona según el Tratado de Luis Lobera de Ávila, siglo XVI". Valencia: Fundación de Enfermería Internacional, 1994.

- A. Martínez Molina, Estudio histórico de la Enfermería en España. Vol. II: "La profesión de la Matrona según el Tratado de Francisco Núnez de Coria, siglo XVI”. Valencia: CECOVA, 1995.

- A. Martínez Molina, Estudio histórico de la Enfermería en España. Los cuidados pediátricos según los Tratados de Materno-infantil del siglo XVI. Valencia: CECOVA, 1995.

- Y. Mastranzo Sánchez y otros, "La enfermera y el cuidado enfermero en una comunidad rural”. En Álvarez Nebreda, Carlos C. y Hernández Martín, F.a J. (editores), El asociacionismo en la Enfermería y su influencia en el desarrollo de la profesión: 100 años de historia del Colegio de Enfermeras de Madrid (18622012). Madrid: Colegio Oficial de Enfermería de Madrid, 2013, pp. 577-584.

- M. ${ }^{a}$ T. Miralles, M.a F.a Casas, "El pistero". Hiades. Revista de Historia de la Enfermería, n. ${ }^{\circ} 10$ (2008), pp. 439-453.

- A. Nogales Espert, "La atención a los peregrinos en el Camino de Santiago: su importancia para la enfermería medieval y de principios de la Edad Moderna". Híades. Revista de Historia de la Enfermería, n.o 8 (2001), pp. 119-130.

- A. Nogales Espert, "Medidas profilácticas contra la peste en Valencia en el año 
1637". Hiades. Revista de Historia de la Enfermería, n. ${ }^{\circ} 10$ (2008), pp. 427438.

- E. Oltra, L. Mendiolagoitia, "Enfermería y Cirugía Menor. Antecedentes históricos". Hiades. Revista de Historia de la Enfermería, n. ${ }^{\circ} 8$ (2001), pp. 441445.

- A. Pedrola Segarra, N. Roca Caparà, "Del Fatebenefratelli de San Juan de Dios a la Teoria del Caring de Jean Watson. Enseñando a nuestros estudiantes a ser cuidadores". Hiades. Revista de Historia de la Enfermería, n.o 11 (2014).

- E. de la Peña, R. Gómez, J. Garrido, S. Galeano, "Los cuidados de la fiebre en la España romana y su proyección hacia la actualidad". Hiades. Revista de Historia de la Enfermería, n. ${ }^{\circ} 10$ (2008), pp. 483-490.

- N. Peralta García, R. Navarro García, E. López Serrano, S. Tenllado Pérez, M. ${ }^{a}$ J. Valderas Sánchez, E. Ruiz Marti, "Historia de la Lepra en Barcelona. Evolución de los cuidados enfermeros". Hiades. Revista de Historia de la Enfermería, n. 11 (2014).

- M. Pérez Luna, "Cuidados enfermeros en el Badajoz del XVII". Hiades. Revista de Historia de la Enfermería, n. 10 (2008), pp. 371-377.

- A. Sánchez Ribes, "Marco de atención al peregrino en los caminos de Santiago". Hiades. Revista de Historia de la Enfermería, n. ${ }^{\circ} 8$ (2001), pp. 57-84.

- M. Santo Tomás Pérez, "Los cuidados vistos a través de la iconografia del Camino de Santiago". Hiades. Revista de Historia de la Enfermería, n. 8 (2001), pp. 99-118.

- M. ${ }^{a}$ J. Sanz Fuentes, "Papel de la mujer como cuidadora en el Camino de Santiago". Hiades. Revista de Historia de la Enfermería, n. 8 (2001), pp. 27-38.

- J. Siles González, "Los cuidados de enfermería en el marco de la historia social y la historia cultural”. En C. González Canalejo, F. Martínez López(editores), $L a$ transformación de la Enfermería. Nuevas miradas para la Historia. Granada: Editorial Comares, 2010, pp. 219-250.

- J. Soldevilla, F. Martínez, "Pasado y presente en el cuidado de las heridas". Híades. Revista de Historia de la Enfermería, n. 8 (2001), pp. 469-478.

- J. I. Valle Racero, M. J. García Martínez, A. C. García Martínez, "Las funciones de enfermería en los Hermanos Obregones (siglos XVI-XVIII). Cuidados y atención de enfermería”. Escuela Universitaria de Enfermería, Fisioterapia y Podología (Universidad Complutense de Madrid), Escuela Universitaria de Enfermería del Hospital General Universitario de Valencia, Libro de Ponencias y Comunicaciones del I Congreso Nacional de Historia de la Enfermería, celebrado en Valencia. Barcelona: Fundación Uriach 1838, 1996, pp. 71-76. 
Fuentes PARA la Historia de la EnFermería

- M. a Mar Alonso, M. Pérez, C. Plaza, "La novela de la segunda mitad del siglo XIX como fuente histórica para la Historia de la Enfermería”. Hiades. Revista de Historia de la Enfermería, n. ${ }^{\circ} 10$ (2008), pp. 827-842.

- M. Amezcua, "La revista Index de Enfermería: Información bibliográfica, investigación y humanidades". Cultura de los Cuidados. Revista de Enfermería y Humanidades, n. ${ }^{\circ}$ /8. Alicante: Seminario de Historia y Antropología de los Cuidados Enfermeros. Departamento de Enfermería. Universidad de Alicante, 2000, pp. 68-74.

- T. Ayuso López y otros, Fuentes documentales sobre el trabajo de las mujeres. Madrid: Ediciones Akal, S. A., 1997.

- J. Bueno y González, Instrucción de novicios de la Orden de la Hospitalidad. Tomo II: El arte de Enfermería (1833). Zaragoza: Consejo Autonómico de Colegios de Enfermería de Aragón, 1997.

- C. C. Álvarez Nebreda, Código Enfermero Español. Siglo XX: Compendio Legislativo. Madrid: Instituto de Salud Carlos III. Ministerio de Sanidad y Consumo, 2002.

- C. C. Álvarez Nebreda, Catálogo Bibliográfico de Publicaciones Enfermeras (15411978). Madrid: Colegio Oficial de Enfermería de Madrid, 2008.

- C. C. Álvarez Nebreda, La Enfermería a través de los diccionarios (1611-2012). Madrid: Colegio Oficial de Enfermería de Madrid, 2012.

- M. A. Calvo Calvo, "Análisis iconográfico de la pintura de Lucas Valdés, Asistencia a los sacerdotes en la enfermeria". Hiades. Revista de Historia de la Enfermeria, n.o 10 (2008), pp. 403-412.

- E. Carmona Samper, M. ${ }^{a}$ I Ruiz García, "Bases de datos para la recuperación de la memoria histórica”. En C. González Canalejo, F. Martínez López (editores), La transformación de la Enfermería. Nuevas miradas para la Historia. Granada: Editorial Comares, 2010, pp. 483-498.

- F. de Castro, Historia de la Vida y Santas Obras de San Juan de Dios. Granada: Casa de Antonio de Lebrixia, 1585.

- A. Delgado Marchante, "Fuentes documentales del Hospital de Santiago de Cuenca recogidas en el Archivo Histórico Nacional”. Hiades. Revista de Historia de la Enfermería, n. ${ }^{\circ} 10$ (2008), pp. 321-330.

- R. Expósito González, "Los manuales para la formación de las enfermeras de la Cruz Roja Espańola”. En C. González Canalejo, F. Martínez López (editores), La transformación de la Enfermería. Nuevas miradas para la Historia. Granada: Editorial Comares, 2010, pp. 311-324.

- I. Fargues i García, R. Tey, "Series documentales referidas a ingresados en el Hospital de la Santa Creu i Sant Pau de Barcelona. Archivo Histórico H. S. C. S. P.”. Hiades. Revista de Historia de la Enfermería, n. 10 (2008), pp. 301-306. 
- I. Fargues i García, "Programa (1922) de la Escuela de Enfermeras de la Mancomunitat de Catalunya en relación con sus contemporáneos". Hiades. Revista de Historia de la Enfermería, n. ${ }^{\circ} 11$ (2014).

- M. ${ }^{a}$ L. Fernández Fernández, M. Santo Tomás, "Arte de cuidar a los enfermos". Hiades. Revista de Historia de la Enfermería, n. 10 (2008), pp. 331-338.

- M. ${ }^{a}$ L. Fernández Fernández, J. L. Callejo, M. Santo Tomás, "El Manual de la enfermera, del doctor Carmelo Valls Marín”. Hiades. Revista de Historia de la Enfermería, n. ${ }^{\circ} 10$ (2008), pp. 339-346.

- M. ${ }^{a}$ L. Fernández Fernández, "La transcendencia del Manual de la Enfermera, de M. Usandizaga, en la construcción del modelo formativo". Hiades. Revista de Historia de la Enfermería, n. ${ }^{\circ} 11$ (2014).

- D. J. Feria Lorenzo, "Los ministrantes ante la Ley de Sanidad de 1855. Análisis de la petición enviada a la Comisión encargada del Dictamen". En C. González Canalejo, F. Martínez López (editores), La transformación de la Enfermería. Nuevas miradas para la Historia. Granada: Editorial Comares, 2010, pp. 295310.

- M. ${ }^{a}$ E. Galiana Sánchez y otros, "El asociacionismo de las enfermeras de salud pública a través de la revista "La Visitadora Sanitaria" (1834-1935)". En Álvarez Nebreda, Carlos C. y Hernández Martín, F.a J. (editores), El asociacionismo en la Enfermería y su influencia en el desarrollo de la profesión: 100 años de historia del Colegio de Enfermeras de Madrid (1862-2012). Madrid: Colegio Oficial de Enfermería de Madrid, 2013, pp. 475-482.

- A. C. García Martínez, "Las Constituciones manuscritas elaboradas por Bernardino de Obregón para la Congregación de los Enfermeros Pobres (¿15921598?)". Hiades. Revista de Historia de la Enfermería, n. 10 (2008), pp. $203-$ 254.

- A. C. García Martínez, "Las publicaciones especializadas de los Practicantes: Un agente de cambio profesional (1857-19369)". En Álvarez Nebreda, Carlos C. y Hernández Martín, F.a J. (editores), El asociacionismo en la Enfermería y su influencia en el desarrollo de la profesión: 100 años de historia del Colegio de Enfermeras de Madrid (1862-2012). Madrid: Colegio Oficial de Enfermería de Madrid, 2013, pp. 91-112.

- A. C. García Martínez y otros, Presentación y análisis de la obra "Instrucción de Enfermeros", de Andrés Fernández, 1625. Aproximación a la Enfermería Española de los siglos XVI-XVII. Madrid: Consejo General de Colegios de Diplomados en Enfermería, 1992.

- A. C. García Martínez, M. J. García Martínez, "Registro y control de las matronas por la Iglesia hispalense. La imagen de la matrona a través de los Libros de Visitas Pastorales del Arzobispado de Sevilla, siglos XVII y XVIII". Hiades. Revista de Historia de la Enfermería, n. ${ }^{\circ} 1$ (1994), pp. 13-33.

- A. C. García Martínez, M. J. García Martínez, J. I. Valle Racero, "Un siglo 
de Oro para la Enfermería española (1550-1650): I. Aparición de Órdenes y Manuales de Enfermería”. En F. Hernández Martín (Coord.): Historia de la Enfermería en España (desde la Antigüedad hasta nuestros dias). Madrid:Editorial Síntesis, S. A., 1996, pp. 137-148.

- A. C. García Martínez, M. J. García Martínez, "Híades, un proyecto para la recuperación de la Historia de la Enfermería". Cultura de los Cuidados. Revista de Enfermería y Humanidades, n. ${ }^{\circ}$ 7/8 (2000), pp. 35-41.

- M. J. García Martínez, "Cultura y alimentación. La dietética en la Enfermería hospitalaria del siglo XVII". Revista Rol de Enfermería, n. ${ }^{\circ}$ 5, vol. 22 (mayo de 1999), pp. 371-381.

- M. J. García Martínez, A. C. García Martínez, "Mil referencias bibliográficas para la Historia de la Enfermería". Hiades. Revista de Historia de la Enfermería, n. ${ }^{\circ} 4$ (1997), pp. 1-123.

- M. J. García Martínez, A. C. García Martínez, "La enseñanza de la Enfermería en la España del siglo XVII. El manual de Enfermería de Simón López (1668)". Cultura de los Cuidados. Revista de Enfermería y Humanidades, n. ${ }^{\circ} 3$ (1998), pp. 15-23.

- M. J. García Martínez, "Religiosidad popular y práctica hospitalaria: su reflejo en la Enfermería espańola del siglo XVII a través de los manuales para la enseñanza de los enfermeros”. En Rodríguez Becerra, S. (coord.), Religión y Cultura. Sevilla: Consejería de Cultura de la Junta de Andalucía y Fundación Machado, 1999.

- M. J. García Martínez, A. C. García Martínez, “Documentos para la Historia de las matronas en América: El proceso de fe contra Juana Prudencia Echavarría. Lima, 1778”. Hiades. Revista de Historia de la Enfermería, n. 10 (2008), pp. 1007-1044.

- M. J. García Martínez, "Cuba: adaptación normativa para el ejercicio de la profesión de Matrona a mediados del siglo XIX". En Álvarez Nebreda, Carlos C. y Hernández Martín, F.a J. (editores), El asociacionismo en la Enfermería y su influencia en el desarrollo de la profesión: 100 años de historia del Colegio de Enfermeras de Madrid (1862-2012). Madrid: Colegio Oficial de Enfermería de Madrid, 2013, pp. 499-506.

- M. ${ }^{a}$ P. García Paramio, Ma E. Galiana Sánchez, J. Bernabeu Maestre, "La Praxis de la Enfermería a través de la revista Enfermeras (1950-1951)". Hiades. Revista de Historia de la Enfermería, n.o 11 (2014).

- E. Gascón, M. ${ }^{a}$ E. Galiana, J. Bernabeu, "Fuentes para el estudio de la Historia Contemporánea de la Enfermería de Salud Pública”. Hiades. Revista de Historia de la Enfermería, n. ${ }^{\circ} 10$ (2008), pp. 353-360.

- R. Gil Sacaluga, "Reglamento general del Hospital Provincial Moreno de Mora de Cádiz, Dispensario-Sifilicomio (1917)". Hiades. Revista de Historia de la Enfermería, n. ${ }^{\circ} 10$ (2008), pp. 373-380. 
- J. L Gómez, D. Ruiz, B. Carrasco, M. a L. Polo, "Análisis de las publicaciones de Historia de la Enfermería: un acercamiento cuantitativo-cualitativo". Hiades. Revista de Historia de la Enfermería, n. 10 (2008), pp. 889-902.

- C. González Guitián, J. Canalejo, N. Sobrido Prieto, "Fuentes para la Historia de la Enfermería Española en Internet". Hiades. Revista de Historia de la Enfermería, n. ${ }^{\circ} 10$ (2008), pp. 921-930.

- J. E. Guerra González, D. J. Feria Lorenzo, "El colectivo de Practicantes, ATS y Enfermeros en el noticiario onubense "Odiel" (1847-1970). Improntas de una profesión”. En C. González Canalejo, F. Martínez López (editores), La transformación de la Enfermería. Nuevas miradas para la Historia. Granada: Editorial Comares, 2010, pp. 373-392.

- J. E. Guerra González, "Historia de la Enfermería. Reflejo en la filatelia mundial". Hiades. Revista de Historia de la Enfermería, n. ${ }^{\circ} 10$ (2008), pp. 781-804.

- J. E. Guerra González, C. Ma Martínez Sánchez, "Iconografía cuidadora en el texto numismático Standard Catalog of Word Coins 1901-2000". Hiades. Revista de Historia de la Enfermería, n. ${ }^{\circ} 11$ (2014).

- F. Herrera Rodríguez, "Reflexiones sobre fuentes documentales para la investigación histórica de la Enfermería". Revista de Humanidades de Jerez (1989), pp. 63-67.

- F. Herrera Rodríguez, J. E. Lasarte Calderay, "La prensa andaluza de los practicantes y matronas (1916-1939)". Comunicación presentada al I Congreso Nacional de Historia de la Enfermería. Valencia, 28-29 de octubre de 1994.

- M. Kenny, J. M. de Miguel, (Editores), La Antropología Médica en España. Barcelona:Anagrama. Biblioteca Anagrama de Antropología, n. ${ }^{o} 15$.

- M. Linares Abad, "Libro de Registro de Matronas, Libro de Actas y Legajos de Hacienda. Fuentes fundamentales para la investigación de género en las profesiones sanitarias". En C. González Canalejo, F. Martínez López (editores), La transformación de la Enfermería. Nuevas miradas para la Historia. Granada: Editorial Comares, 2010, pp. 469-482.

- S. López, Directorio de Enfermeros y artífice de obras de caridad para curar las enfermedades del cuerpo. Biblioteca de Clásicos de la Enfermería Española, n. ${ }^{\circ}$ 1. Estudio, trascripción e índices: Antonio C. García Martínez y Manuel J. García Martínez. Sevilla: Consejo General de Enfermería de España, 2001.

- I. M. a López, V. Sánchez, "Producción científica sobre Historia de la Enfermería en España". Hiades. Revista de Historia de la Enfermería, n. ${ }^{\circ} 10$ (2008), pp. 943-955.

- M. ${ }^{a}$ J. López Medina, "Fuentes para el estudio de las cuidadoras en época romana: los agónomos latinos”. En C. González Canalejo, F. Martínez López (editores), La transformación de la Enfermería. Nuevas miradas para la Historia. Granada: Editorial Comares, 2010, pp. 55-74.

- J. M. ${ }^{a}$ López Piñero, M. ${ }^{a}$ L. Terrada Ferrandis, Información cientifica en me- 
dicina y sus fuentes. Valencia: Servicio de Publicaciones de la Universidad de Valencia, 1993.

- C. Lousada, M. Vieira, "Manuais para as Práticas dos enfermeiros no Antigo Regime". Hiades. Revista de Historia de la Enfermería, n. ${ }^{o} 11$ (2014).

- S. Luna Gálvez, "La Enfermería en la Filatelia". Hiades. Revista de Historia de la Enfermería, n. 10 (2008), pp. 465-499.

- M. ${ }^{a}$ T. Miralles Sangro, "La filatelia como fuente para el estudio iconográfico de la Historia de la Enfermería. La imagen como testigo". En C. González Canalejo, F. Martínez López (editores), La transformación de la Enfermería. Nuevas miradas para la Historia. Granada: Editorial Comares, 2010, pp. 403-414.

- M. ${ }^{a}$ T. Miralles Sangro, "De la colección al Museo”. En Álvarez Nebreda, Carlos

C. y Hernández Martín, F.a J. (editores), El asociacionismo en la Enfermería y su influencia en el desarrollo de la profesión: 100 años de historia del Colegio de Enfermeras de Madrid (1862-2012). Madrid: Colegio Oficial de Enfermería de Madrid, 2013, pp. 339-346.

- P. Moral de Calatrava, "Espacios femeninos. El proceso de legitimación masculina del saber y las prácticas sanitarias femeninas (siglos XIII-XVI)”. En C. González Canalejo, F. Martínez López (editores), La transformación de la Enfermería. Nuevas miradas para la Historia. Granada: Editorial Comares, 2010, pp. 75-94.

- J. Siles y otros, "La Enfermería a través del cine: un estudio histórico y transcultural". Hiades. Revista de Historia de la Enfermería, n. 10 (2008), pp. 761780.

- A. Nogales Espert, "El asociacionismo en el arte de la Enfermería. Hacia una estética del optimismo: la ilusión”. En Álvarez Nebreda, Carlos C. y Hernández Martín, F.a J. (editores), El asociacionismo en la Enfermería y su influencia en el desarrollo de la profesión: 100 años de historia del Colegio de Enfermeras de Madrid (1862-2012). Madrid: Colegio Oficial de Enfermería de Madrid, 2013, pp. 275-296.

- M. C. Palacín Gálvez, L. Martínez García, Documentación del Hospital del Rey de Burgos. Fuentes medievales castellano-leonesas. Burgos: 1990.

- J. Parrilla y otros, "Fuentes documentales actuales dirigidas a facilitar el desarrollo científico de las enfermeras". Hiades. Revista de Historia de la Enfermería, n. ${ }^{\circ} 10$ (2008), pp. 381-394.

- A. Pedraz Marcos, A. M. a Palmar Santos, A. García González, "Estudio de las fuentes en el aprendizaje de la Historia en el Grado de Enfermería". En Álvarez Nebreda, Carlos C. y Hernández Martín, F.a J. (editores), El asociacionismo en la Enfermería y su influencia en el desarrollo de la profesión: 100 años de historia del Colegio de Enfermeras de Madrid (1862-2012). Madrid: Colegio Oficial de Enfermería de Madrid, 2013, pp. 605-612.

- J. Siles, "Fuentes etnográficas e Historia de la Enfermería". Hiades. Revista de 
Historia de la Enfermería, n.o 10 (2008), pp. 111-138.

- S. Trías Mercant, "Los documentos y la cultura material". Aguirre (Ed.), Etnografía. Metodología cualitativa en la investigación sociocultural. Barcelona: Editorial Boixareu Macombo, 1995, pp. 160-170.

- T. Uliarte, L. Campos, "El sello postal como fuente de información de los cuidados de las enfermeras en las grandes pandemias". En C. González Canalejo, F. Martínez López (editores), La transformación de la Enfermería. Nuevas miradas para la Historia. Granada: Editorial Comares, 2010, pp. 415-446.

- A. Urmeneta Marín, "Intervención de una comadrona en el peritaje de un caso de impotencia femenina en Estela (Navarra), año 1650". Híades. Revista de Historia de la Enfermería, n. ${ }^{\circ} 10$ (2008), pp. 997-1006.

- J. I. Valle Racero, "La revista "El Practicante Sevillano" en el período convulso de 1931 a 1936". Hiades. Revista de Historia de la Enfermería, n. ${ }^{\circ} 10$ (2008), pp. 361-372.

- R. Vallespin y otros, "Fuentes para la investigación de la Historia de la Enfermería en Gran Canaria". Hiades. Revista de Historia de la Enfermería, n. ${ }^{\circ} 10$ (2008), pp. 395-402.

Publicaciones periódicas CON MAYOR PRESENCIA DE LA Historia de LA ENFERMERÍA

- Cultura de los Cuidados. Revista de Enfermería y Humanidades (Universidad de Alicante).Web: www.culturacuidados.ua.es

- Hiades. Revista de Historia de la Enfermería (Qalat Chábir, A. C. Alcalá de Guadaíra. Sevilla).Web: www.portalhiades.com

- Índex de Enfermería (Fundación Index. Granada).

Web: www.index-f.com/index-enfermeria/revista.php

- Temperamentum. Revista Internacional de Historia y Pensamiento enfermero (Fundación Index. Granada).

Web: www.index-f.com/temperamentum/revista.php

Libros de Actas de Congresos de Historia de la Enfermería celebraDOS EN ESPAÑA

- Escuela Universitaria de Enfermería, Fisioterapia y Podología (Universidad Complutense de Madrid), Escuela Universitaria de Enfermería del Hospital General Universitario de Valencia, Libro de Ponencias y Comunicaciones del I Congreso Nacional de Historia de la Enfermería, celebrado en Valencia. Barcelona: Fundación Uriach 1838, 1996.

- Escuela Universitaria de Enfermería, Fisioterapia y Podología (Universidad Complutense de Madrid), Escuela de Ciencias de la Salud (Universidad de 
Málaga), "La Enfermería en España desde sus orígenes hasta la Edad Media". Libro de Actas del II Congreso Nacional de Historia de la Enfermería, celebrado en Málaga. Jaén: 1998.

- Escuela Universitaria de Enfermería de Mérida,"El cuidado, pilar de la asistencia sanitaria a través de la Historia”. Libro de Ponencias y Comunicaciones del III Congreso Nacional de Historia de la Enfermería, celebrado en Mérida. Barcelona: Excma. Diputación Provincial de Badajoz, 1999.

- Hiades. Revista de Historia de la Enfermería, n.o 8,"La Enfermería en las Rutas Jacobeas: perspectiva histórica". Libro de Actas del IV Congreso Nacional de Historia de la Enfermería, celebrado en Gijón durante los días 6 al 8 de mayo de 1999. Alcalá de Guadaíra (Sevilla): Qalat Chábir, A. C., 2001.

- Hiades. Revista de Historia de la Enfermería, n. ${ }^{\circ}$ 10,"Tradición y Modernidad. Las fuentes para el estudio de la Historia de la Enfermería”. Libro de Actas del $V$ Congreso Nacional de Historia de la Enfermería, celebrado en Sevilla durante los días 25 al 27 de octubre de 2001. Alcalá de Guadaíra (Sevilla): Qalat Chábir, A. C., 2008.

- Escuela de Enfermería y Fisioterapia (Universidad de Alcalá),Libro de Actas del VI Congreso Nacional y I Internacional de Historia de la Enfermería, celebrado en Alcalá de Henares del 3 al 5 de abril de 2003. Navarra: Servicio de Publicaciones de la Universidad de Alcalá, 2003.

- Diputación Provincial de Zaragoza, "Cuidadoras en la Historia: Protagonistas de ayer y de hoy". Libro de Actas del III Congreso Internacional y VIII Nacional de Historia de la Enfermería, celebrado en Zaragoza del 27 al 29 de octubre de 2005. Zaragoza: Servicio de Publicaciones de la Diputación Provincial de Zaragoza, 2007.

- C. González Canalejo, F. Martínez López(editores),La transformación de la Enfermería. Nuevas miradas para la Historia. X Congreso Nacional y V Internacional de Historia de la Enfermería celebrado en Almería en noviembre de 2008. Granada: Editorial Comares, 2010.

- Hiades. Revista de Historia de la Enfermería, n. ${ }^{\circ} 11$, "La influencia de la Historia en la construcción del pensamiento enfermero". Libro de Actas del XI Congreso Nacional y VI Internacional de Historia de la Enfermeria, celebrado en Barcelona durante los días 25 al 27 de noviembre de 2010. Alcalá de Guadaíra (Sevilla): Qalat Chábir, A. C., 2014.

- Álvarez Nebreda, Carlos C. y Hernández Martín, F.a J. (editores), El asociacionismo en la Enfermería y su influencia en el desarrollo de la profesión: 100 años de historia del Colegio de Enfermeras de Madrid (1862-2012). VIII Congreso Internacional y XIII Nacional de Historia de la Enfermería celebrado en Madrid en 2012. Madrid: Colegio Oficial de Enfermería de Madrid, 2013. 
